\title{
Modular localization and the foundational origin of integrability
}

\author{
dedicated to Raymond Stora on the occasion of his 80th birthday \\ Bert Schroer \\ present address: CBPF, Rua Dr. Xavier Sigaud 150, \\ 22290-180 Rio de Janeiro, Brazil \\ email schroer@cbpf.br \\ permanent address: Institut für Theoretische Physik \\ FU-Berlin, Arnimallee 14, 14195 Berlin, Germany
}

November, 2012

\begin{abstract}
The main aim of this work is to relate integrability in QFT with a complete particle interpretation directly to the principle of causal localization, circumventing the standard method of finding sufficiently many conservation laws. Its precise conceptual-mathematical formulation as "modular localization" within the setting of local operator algebras also suggests novel ways of looking at general (non-integrable) QFTs which are not based on quantizing classical field theories.

Conformal QFT, which is known to admit no particle interpretation, suggest the presence of a "partial" integrability, referred to as "conformal integrability". This manifests itself in a "braid-permutation" group structure which contains in particular informations about the anomalous dimensional spectrum. For chiral conformal models this reduces to the braid group as it is represented in Hecke- or Birman-Wenzl- algebras associated to chiral models.

Another application of modular localization mentioned in this work is an alternative to the BRST formulation of gauge theories in terms of stringlike vectorpotentials within a Hilbert space setting.
\end{abstract}

\section{Integrability in classical and quantum theory}

The notion of integrability arose in celestial mechanics and referred to systems for which the equation of motion can be solved in closed analytic form without the necessity to resort to controlled approximations (perturbation theory). The prototype model was the Kepler two-body system, whereas more than two celestial bodies lead to non-integrable situations which can only be approximated 
(in principle with unlimited numerical accuracy). For the non-integrable case the terminology does not just mean that no analytic solution was found, but rather points to the existence of a proof that such a solution does not exist.

As the mathematical sophistication evolved, physicists and mathematicians developed model-independent criteria for integrability. A modern definition which is sufficiently general to cover classical mechanics is in terms of a complete set of conservation laws in involution [1].

This definition was extended from mechanics to include classical field theory where, according to Noether's theorem, a symmetry in the Lagrangian setting leads to a conserved current and integrability means that there exists an infinite complete set of conserved currents in involution. Quantum mechanics is basically what is obtained from classical mechanics by "quantization"; the fact that this process is not an isomorphism but a more artistic kind of correspondence (the problem of ordering of operator products) did not affect the inference of quantum integrability via quantization from its classical counterpart. The best known illustration is the quantum analog of the Kepler problem i.e. the hydrogen atom. In this case the conservation laws which lead to integrability can be elegantly presented in terms a spectrum-setting $O(4,2)$ group symmetry. Anomalies which could prevent conservation laws to be inherited from their classical counterpart usually need the presence of infinitely many degrees of freedom and hence occur predominantly in quantum field theory (QFT).

There are many models of QFT which have remained outside the range of Lagrangian quantization because no Lagrangian which fits them has been found; in particular most of the so-called $\mathrm{d}=1+1$ factorizing models, for which explicit expressions for formfactors of quantum fields were constructed within the bootstrap-formfactor program remained without a classical Lagrangian name. Their given name refers to internal symmetries or to analogies with lattice models. An example for such a situation is the scaling $\mathrm{Z}(\mathrm{N})$ Ising model in [2], as the authors emphasize in the introduction of their paper. Factorizing models constitute a nontrivial class of models with generally noncanonical short distance behavior which owe their existence proof to operator-algebraic methods. These methods are significantly different from the quantum mechanical and measure-theoretical functional methods used by Glimm and Jaffe [3] in the 60s for establishing the existence of certain canonical (superrenormalizable) models in the Lagrangian setting; in fact the new method is based on modular localization [4].

Among the few integrable QFT which allow a Lagrangian presentation, the Sine-Gordon model is the most prominent. The first indication about its integrability came from the famous quasiclassical observations on the Sine-Gordon particle spectrum by Dashen-Hasslacher and Neveu [5]. But even in cases like this, where the Lagrangian quantization setting even provides a renormalized perturbation series, the latter still carries the stain of divergence of all QFT perturbation series; Lagrangian quantization "baptizes" a model with a name from classical field theory and permits a perturbative expansion, but it does not lead to a proof of the existence of a QFT behind this formalism, so that the problem of mathematically controlled approximations cannot even be formulated. 
Despite the observational success of the lowest terms in powers of the coupling strength, one does not even know whether the power series is at least an asymptotic approximand in the limit of vanishing coupling; the numerical successes of renormalized QED and the subsequent observational achievements of the standard model have no direct bearing on the mathematical consistency of what is being approximated. Compared with other areas of theoretical physics this is quite unique and shows, that in spite of almost 90 years which passed since it was discovered, QFT still is not anywhere near its closure.

Recent insights into integrable $\mathrm{d}=1+1$ factorizing models did not result from refinements of the classical parallelism of the Lagrangian- or functional- quantization setting. Rather it has been revealed through representation theory, a method which was first introduced in 1939 by Wigner as a means to obtain an intrinsic systematic classification of wave function spaces of relativistic particles instead of having to cope with an ever-growing confusing zoo of field equations, many leading to equivalent descriptions. In the context of QFT, representation theoretical methods were first used in connection with current algebras and chiral conformal QFTs. The discovery of integrable QFTs with a particle interpretation started with Dashen-Hasslacher-Neveu's [5] quasiclassical observations about the Sine-Gordon particle spectrum and took an interesting turn after it was realized that it could be viewed as an exact realization of the "nuclear democracy" particle spectrum which originated in the bootstrap setting of scattering functions in $\mathrm{d}=1+1[6]$. The second important step was the discovery of the representation theoretical bootstrap-formfactor setting [7]. This project reached its present perfection after it was realized that:

- Unitary and crossing symmetric elastic two-particle scattering functions which obey the Yang-Baxter consistency relations can be classified [9][10] and lead to combinatorial factorization formula for n-particle elastic Smatrices. Zamolodchikov's formal algebraization added a useful tool [12] to the implementation of this (originally analytic) classification project.

- The bootstrap-formfactor program associates to each such scattering function $^{1}$ explicitly computable formfactors of local covariant fields from the local equivalence class (Borchers class) of (composite) fields. The relation of the scattering function to an associated QFT is unique (uniqueness of the solution of the inverse scattering problem within the bootstrapformfactor setting).

- The creation and annihilation operators of the Zamolodchikov-Faddeev (ZF) algebra turned out to be the Fourier components of covariant vacuumpolarization-free generators (PFGs) of interacting wedge-localized algebras [14][15]. They are special interacting operators within the theory of "modular localization" which map the vacuum into a one-particle state i.e. they permits to "emulate" a wedge-smeared incoming field in terms of an

\footnotetext{
${ }^{1}$ In the presence of backward scattering and/or inner symmetry indices the scattering function is a matrix function which fulfills the Yang-Baxter equation [13].
} 
operator affiliated to the wedge-localized operator algebra. This emulation construction has a generalization to multiparticle incoming fields.

- The action of translations on a wedge-localized algebra together with that of the modular reflection (in $\mathrm{d}=1+1$ the TCP operation) generate a net of right and left directed wedge algebras whose double cone intersections are compactly localized algebras which act cyclic and separating on the vacuum [16][4]. In these papers the new constructive setting based on modular localization obtained its first mathematical formulation. Combined with the control of phase space degrees of freedom in the form of modular nuclearity, this approach led to the first existence proof of QFTs with non-canonical short distance behavior within the algebraic setting of QFT.

- A sharp division between temperate and non-temperate vacuum-polarizationfree generators (PFGs) of wedge-localized algebras gives rise to a dichotomy of integrable and non-integrable QFTs.

The integrable PFGs with their Z-F algebraic structure, apart from being nonlocal (i.e. wedge- instead of point- local), relate particles and fields in the standard way. Non-integrable PFGs on the other hand have translation non-invariant domain properties which are radically different from those which one meets in the standard formulation of fields or even with operators used in nonlocal or noncommutative extensions [18]. The much weaker relation of particles with localized algebras and fields resulting from non-temperate PFGs accounts for the difficulties one faces in studying non-integrable models of QFT. It explains in particular why even 8 decades after its discovery, a mathematically controlled construction of non-integrable models of QFT remaines an open problem [19]. Although our presentation in section 5 will not solve these problems, it does try to place them into sharper focus which may be useful for future constructive attempts.

The DHR superselection theory [21], which constructs a full QFT from its local observables algebras, leads to a different kind of "partial" integrability which is of a more kinematical kind. In theories which are non-integrable in the previous (dynamical) sense, the superselection strucure of observable algebras can be described in terms of an exactly computable combinatorial algebraic structure, even though the observable algebra itself remains non-integrable. With some hindsight one can find out which integrable algebraic superselection structure belongs to a specific non-integrable net of local observable algebras. Since Lagrangian quantization leads directly to the perturbation theory of the full field algebra (whose fixed-point algebra under a compact inner symmetry group action defines the observable algebra), this distinction between observables and their superselection structure is mainly of conceptual, but hardly of practical interest.

The situation becomes more interesting for conformal QFT, in which case the separation of observables from the superselection charge-carrying fields arises in a natural way through the Huygens principle, according to which observables 
commute for space- and time-like separations. In the context of QFT this limits the notion of observable algebras to algebras which are generated by pointlike fields with integer scale dimension, whereas fields which carry anomalous dimensions are considered as superselected charge carriers similar to the DHR superselection of massive QFT. This attaches to the anomalous dimensions a "Huygens superselection" aspect.

Indeed, the anomalous scale spectrum appears in the spectrum of the center of the universal covering of the conformal group, and unlike the anomalous spin spectrum in the Bargman-Wigner representation theory of the Lorentz group in $\mathrm{d}=1+2$, it is accompanied by the geometric covering of the (compactified) Minkowski space. The latter consists of infinitely many "heavens" above and "hells" below [22] and hence the anomalous dimensions which control the short distance behavior also show up in phase factors which arise from the conformal rotation in passing from one Minkowski spacetime copy to the next.

In an appropriately extended terminology the anomalous dimensions together with the braid-permutation group $\mathbf{B} \mathbf{P}_{\infty}$ [23] structure belong to the kinematical aspects of a conformal theory. In $\mathrm{d}=1+1$ the conformal group and the observable algebra split into right and left parts and the $\mathbf{B} \mathbf{P}_{\infty}$ group simplifies and becomes the braid group $\mathbf{B}_{\infty}$, whereas the observable algebras (current, energy-momentum) reduce to well-studied infinite Lie-algebras. The combinatorial braid group algebra has interesting connections with Vaughn Jones [24] mathematical work on subfactors. In the last section this situation will be presented in more details.

The natural setting for the modular theory is that of local quantum physics (LQP) [21] which appeared in a rudimentary form already in 1957 in Haag's first attempt to formulate QFT in an intrinsic way [25] i.e. without leaning on the parallelism to the classical world of Lagrangian fields. This formulation competed with the (at that time already existing) framework by Wightman [26] in which for the first time quantum fields were identified with operator-valued Laurent Schwartz distributions. The understanding of the inherently singular behavior of quantum fields as compared to their classical counterparts was the key for "taming" the ultraviolet divergence problem, which before almost caused the abandonment of QFT. Haag's LQP approach attributes to these fields the role of (singular) generators of a net of localized operator algebras; another helpful analogy is that to coordinatizations in geometry.

Both settings were strongly influenced by Wigner's 1939 particle classification in terms of group representation concepts for the inhomogeneous Lorentz group (the Poincaré group $\mathcal{P}$ ) as an alternative to the quantization of classical field equations. Haag's basic idea was simple and almost naive: measurements of local observables in a spacetime region $\mathcal{O}$ which have a certain duration in time (the duration of the activation of a particle counter) and a spatial extension within $\mathcal{O}$ should be members of an ensemble of operators forming an operator algebra $\mathcal{A}(\mathcal{O})$. An experimentalist does not have to know the internal structure of his particle- and radiation- counters, his only means to increase precision is to improve their spacetime localizing sensitivity as well as using several of them in coincidence and anti-coincidence arrangements. For extracting scattering data 
from QFT it is not necessary to know the detailed properties of an individual $\mathcal{O}$-localized observable, the information that it belongs to a localized ensemble $\mathcal{A}(\mathcal{O})$ and to identify its superselected charge suffices [21].

The first test of this idea came from the derivation of the Lehmann-SymanzikZimmermann (LSZ) scattering formula from the large-time behavior of operators used in scattering theory in which only their affiliation to a localized ensemble is used; differences between individual operators in $\mathcal{A}(\mathcal{O})$ only show up in adjustable numerical normalization factors of their asymptotic limits (the insensitivity of the S-matrix against local changes [21]). This viewpoint of placing ensembles in form of localized operator algebras into the center stage has shown its soundness in numerous special situations; last not least it accounts for the fact that there is no necessity for adding the concept of probability to QFT since, in contrast to QM to which Born had to add the probability interpretation for individual events, the "ensemble" of operators in $\mathcal{A}(\mathcal{O})$ has the properties of a KMS state in statistical mechanics, including the probabilistic aspects of thermal states (apart from the fact that the modular Hamiltonian is not that of time-evolution). In fact it is a consequence of the mathematical description of causal localization in the form of modular localization which is the foundational principle of LQP.

Born's spatial localization in terms of projectors from the spectral decomposition of the selfadjoint position operator leads to a localization of states for which the probability interpretation has to be added; in QM there is no intrinsic ensemble aspect from localization. This is radically different in LQP where the restriction of the vacuum to a spacetime localized algebra $\mathcal{A}(\mathcal{O})$ acquires a thermal manifestation which the global vacuum as an expectation value on global observables did not have [19]. In mathematical terms, the restriction of the pure global vacuum to localized variables is an impure thermal state to the causally closed "world" associated with a local subalgebra of observables. This phenomenon is best described in the setting of modular operator theory which among other things attaches a modular Hamiltonian $H_{m o d}$ which is uniquely determined by the pair $(\mathcal{A}(\mathcal{O}), \Omega)$ where $\Omega$ denotes the global vacuum. The restricted vacuum is a thermal state which obeys the KMS ${ }^{2}$ relation with respect to $H_{\text {mod }}$. This property shows most clearly the conceptual rift between QM and QFT.

In the Einstein-Jordan conundrum Einstein and Jordan [19] came close to this insight; the observation that Jordan's subvolume fluctuations in QFT are indistinguishable from Einstein's thermal black body system as a consequence of "localization-thermality" which results from the restriction of the pure vacuum state could have clinched the conundrum; but the situation in 1925 was too much under the spell of the conceptual revolution caused by the freshly discovered QM to permit the perception of this subtle difference; so its full understanding had to await more than 8 decades. The phenomenon of vacuum polarization, which was discovered some years later by Heisenberg, has a strong relation to the E-J

\footnotetext{
${ }^{2}$ The KMS relation is an anytic relation which is fulfilled by tracial Gibbs states. It survives in the thermodynamic limit when the tracial characterization is lost [21].
} 
conundrum $^{3}$ since the formation of vacuum polarization clouds at the boundary of a localization region (leading to area proportionality of localization entropy) and the thermal property of the reduced vacuum state are two sides of the same coin.

There can be no doubt that Einstein, who had a livelong philosophical problem with Born's assignment of probabilities to individual events in QM, would have embraced the the ensemble probability from thermalization of a subvolume restricted vacuum state in QFT. After all it was the subvolume fluctuations in the statistical mechanics at finite temperature which led him to the corpuscular aspect of light [19]. But time was not yet ripe to understand that subvolume restriction in QFT in constrast to QM lead to a loss of purity of the restricted vacuum state which results in thermal behavior without a "heat bath" being present.

The existence of a thermal manifestation of causal localization is an unavoidable consequence of Haag's quantum adaptation of the Faraday-Maxwell "Nahewirkungsprinzip" [21] (together with Einstein's refinement of causality in Minkowski spacetime). In fact causal locality and its more recent mathematical formulation as modular localization became the cornerstone of Haag's local quantum physics (LQP) and the thermal manifestation of the subvolume restricted vacuum is a consequence.

The perception of the presence of this intrinsic ensemble probability could have vindicated Einstein's philosophical resistance against Born's probability, so that many physicists nowadays would not think of Einstein's reluctance as being the stubborn resistence of an old man against the tides of the time. Einstein may have even accepted Born's assignment of probability to individual events if it were possible to show, that within a better future understanding of the limiting relation of QM with the more fundamental local QFT, the probabilistic manifestation of a localized ensemble passes to Born's quantum mechanical probability even though causal localization and its epiphenomena vacuum polarization and the KMS property of restricted vacuum STATE disappears in such a limit.

As most ideas which do not result from the extension of an already existing formalism but rather emerged from philosophical contemplations about physical principles, their content is usually more subtle than that of the supporting intuitive arguments. Already for the localization inside a non-compact spacetime region as big as a Rindler wedge only a uniform acceleration in the wedge direction can keep a particle counter (observer) inside a non-compact wedge region (the Unruh Gedankenexperiment [27]); and when it comes to the localization inside a compact causally closed region (e.g. the double cone completion of a spatial ball) it becomes impossible in models with a mass-gap to visualize the realization of modular localization in spacetime geometric/physical terms since

\footnotetext{
${ }^{3}$ In fact in a private correspondence Heisenberg challenged Jordan to account for a logarithmic divergence resulting from the vacuum polarization cloud at the endpoints of his localization interval [20]
} 
the region preserving modular group becomes an abstract automorphism of the localized operator algebra.

The thermal manifestation of modular localization is an unavoidable consequence of Haag's adaptation of classical causality (Maxwell equations) to the requirements of quantum theory, and hence it is fully present in QFT. The impurity of the state resulting from the restriction of the pure global vacuum state to the ensemble of observables contained in a localized (without loss of generality causally closed) algebra and its description in terms of a KMS state associated with a "modular Hamiltonian" is a mathematical fact, notwithstanding the difficulties to find direct observational verifications ${ }^{4}$ which underlines the subtlety of causal localization in QT.

It is not important that physical principles can be directly verified in terms of existing hardware or whether their intuitive support under closer conceptual scrutiny appears somewhat metaphoric; important is primarily the mathematical precision of the formulation and the wealth of the theoretical and observational consequences. For the Unruh Gedankenexperiment, the dependence of the state of the observer attributes a certain aura of fleetingness, but this is to some extend overcome if the horizon is an intrinsic property of the spacetime metric in form of an event horizon. The curvature contained in the spacetime metric does not create the thermal aspects of Hawking radiation, it rather replaces the observer-dependent fleeting aspect of the causal horizon by a more robust observer-independent event horizon and in this way favors the macroscopic detection of localization-thermality in astrophysical observations.

In contrast to the volume-proportional heat bath entropy, the localization entropy of quantum matter is proportional to the dimensionless area $A / \varepsilon^{2} \rightarrow$ $\infty, \varepsilon \rightarrow 0$ where $\varepsilon$ is the surface "roughness" i.e. the thickness of the layer which is conceded to the attenuation of the vacuum polarization cloud. In the Bekenstein conjecture the finite black hole entropy (formally obtained by replacing the parameter $\varepsilon$ by the Planck distance) refers to the hypothetical degrees of freedom of a future quantum theory of gravity. We believe that this is related to 't Hooft's brickwall picture [28]. Such a surface divergence in the limit of sharp localization appeared for the first time in Heisenberg's treatment of vacuum polarization caused by localization of conserved (dimensionless) charges [19].

It is not surprising that the subtlety of the principle of causal localization has led to misunderstandings in particular in string theory (last section). Although the correction of deep errors stemming from misunderstandings is a powerful method to highlight the importance of a principle, we will not follow such a path here and instead refer to other publications [29][30][80].

There remains the question why these important localization properties of QFT have not been seen already long ago. The answer is that they did not play an important role in the Tomonaga-Schwinger-Feynman-Dyson discovery of renormalized perturbation theory; the essential step from the old perturba-

\footnotetext{
${ }^{4}$ There is an ongoing discussion whether thermal radiation effects in Unruh situations can be seen in subnuclear laboratory experiments.
} 
tion theory (in textbooks by Heitler and Wentzel), which had insurmountable problems with vacuum-polarization, to the modern formulation was the implementation of a covariant formulation. Covariance under the Poincaré automorphisms of Minkowski spacetime is closely related to causal localization, but it generally leads to stronger results.

The sections 2 and 4 prepare the ground for "modular localization" which is used in section 5 for a characterization of integrability/non-integrability in terms of properties of generators of wedge-localized operator algebras. Since modular localization is a quite subtle recent concept, and not every reader is prepared to invest in conceptual/mathematical ideas without some indication about their physical relevance for important open problems of particle physics, it may be helpful to indicate what one hopes to achieve from a reformulation of the BRST gauge theory in terms of string-localized vectorpotentials (section 3 ); this new view may even lead to revisions of the Higgs issue.

\section{The modular localization approach of QFT}

There are two routes to modular localization, a mathematical access and a more physical-conceptual path. The mathematical route starts from the TomitaTakesaki modular theory of operator algebras and makes contact with QFT by applying this to Haag's LQP algebraic formulation of QFT in terms of spacetime-indexed nets of operator subalgebras [21]. An important step was the recognition by Bisognano and Wichmann [31][32] that the abstract TomitaTakesaki modular group $\Delta_{\mathcal{A}}^{i t}$ and the modular reflection $J$ acquire a direct geometric-physical meaning in terms of particle physics concepts in case of a wedge-localized operator subalgebra ${ }^{5} \mathcal{A}(W)$, whereas the modular objects for compact localized algebras $\mathcal{A}(\mathcal{O})$ can be in principle determined by representing the region of interests (rather its causal completion) and the associated algebras using intersections of wedges and wedge-localized algebras [33].

Modular theory began in the middle of the $60 \mathrm{~s}$ as a joint venture between mathematics and physics when, at a conference in Baton Rouge [8], mathematician interested in operator algebras (Kadison, Tomita, Takesaki) met physicists (Haag, Hugenholz, Winnink) who just had finished work on an intrinsic formulation of statistical quantum mechanics of open systems which avoids the non-covariant box-quantized Gibbs states by starting directly in the thermodynamic infinite volume limit [21].

In this work they used an older analytic trick from Kubo, Martin and Schwinger to avoid computing Gibbs traces. In the open system setting this acquired a fundamental conceptual significance; in this way the KMS property became part of the joint mathematics/physics heritage, combining modular theory of operator algebras (where it led to Connes famous classification work about von Neumann algebras) with statistical mechanics of open systems. Whereas the box-quantized thermal Gibbs states always stay within the setting of the

\footnotetext{
${ }^{5}$ A general wedge results from $W_{0}=\left\{\left|x_{0}\right|<x_{1}\right\}$ by applying Poincaré transformations.
} 
standard (type $\mathrm{I}_{\infty}$ ) quantum mechanical algebras $B(H)$ of all bounded operators on a Hilbert space, the thermodynamic limit ${ }^{6}$ changes the algebraic type into what afterwards in Connes classification was called the unique hyperfinite type III $_{1}$ von Neumann factor algebra.

At the beginning of the 60s Araki [21] had already shown that such algebras, which have quite different properties from those met in QM, occur in the form of local operator algebras in QFT. Together with the statistical work on open systems this was suggestive of an conceptual connection of thermal behavior and localization but at that time this remained unnoticed. It came as quite a surprise when a decade afterwards Bisognano and Wichmann [31] discovered that the monad $^{7}$, realized as a wedge-localized subalgebra $\mathcal{A}(W)$, has modular data which have well-known physical/geometrical significance, including the KMS property. As a result it became clear that localization, thermalization, and the generation of vacuum polarization clouds are inexorably intertwined.

Using the representation theoretical access to modular localization, we begin our presentation with extracting modular objects from Wigner's classification of irreducible positive energy representations of the Poincaré group. This concept was not available to Wigner who realized that the Born localization based on the position operator to the relativistic inner product (the Newton-Wigner localization) was not the right concept which could connect his particle representation theory with QFT. This may explain why Wigner, after his important contribution to QFT immediately after its discovery, maintained a lifelong critical distance with respect to its later developments. Whereas the Born localization is extrinsic ${ }^{8}$ to QM, the modular localization and its thermal-probabilistic aspect is intrinsic, i.e. it only uses concepts from the representation theory of the Poincaré group. For matters of notational simplicity we restrict our presentation to the case of a scalar massive particle.

It has been realized, first in a special context in [14] and then in a general mathematical rigorous setting which covers all positive energy representations in [35] (see also [36][37]), that there exists a natural localization structure on the Wigner representation space for any positive energy representation of the proper Poincaré group. The starting point is an irreducible $(m>0, s=0)$ one-particle representation of the Poincaré group on a Hilbert space $H^{9}$ of wave functions with the inner product

$$
\left(\varphi_{1}, \varphi_{2}\right)=\int \bar{\varphi}_{1}(p) \varphi_{2}(p) \frac{d^{3} p}{2 p_{0}}
$$

\footnotetext{
${ }^{6}$ The tensor factorization of type $\mathrm{I}_{\infty}$ "thermofield theory" breaks down and the algebra changes its type.

${ }^{7}$ The short name which we will use for the (up to isomorphism unique) befor-mentioned operator algebra. Besides the standard algebra $B(H)$ of all bounded operators this the only type of operator algebra which one encounters in continuous quantum systems [57].

${ }^{8}$ Born localization entered QM through his famous probabilistic interpretation of (the Born approximation of) the scattering amplitude i.e. the cross section. This was afterwards extended to the position operator and its associated wave functions. .

${ }^{9}$ Since positive energy representations are completely reducible this works for all such representations, not only irreducible ones.
} 
For other (higher spin, massless) representations the relation between the momentum space wave function on the mass shell (or light cone) and the covariant wave functions is more involved as a consequence of the presence of intertwiners $u(p, s)$ which connect the unitary with the covariant representations. Selecting a wedge region $W_{0}=\left\{x \in \mathbb{R}^{d}, x^{d-1}>\left|x^{0}\right|\right\}$ one notices that the unitary wedgepreserving boost $U\left(\Lambda_{W}(\chi=-2 \pi t)\right)=\Delta^{i t}$ commutes with the antiunitary reflection $J_{W}$ on the edge of the wedge (i.e. along the coordinates $x^{d-1}-x^{0}$ ). The distinguished role of the wedge region is that it produces a commuting pair of boost and antiunitary reflection. This has the unusual (and perhaps even unexpected) consequence that the closed, antiunitary operator (the Tomita Soperator),

$$
\begin{array}{r}
S_{W}:=J_{W} \Delta^{\frac{1}{2}}, \quad S_{W}^{2} \subset 1 \\
\text { since } J \Delta^{\frac{1}{2}} J=\Delta^{-\frac{1}{2}}
\end{array}
$$

which is intrinsically defined in terms of Wigner representation data, is involutive on its dense domain and has a unique closure (unchanged notation) with $\operatorname{ranS}=$ dom $S$.

The involutivity means that the s-operator has \pm 1 eigenspaces; since it is antilinear, the + space multiplied with $i$ changes the sign and becomes the space; hence it suffices to introduce a notation for just one eigenspace

$$
\begin{gathered}
K(W)=\left\{\psi \text { in domain of } \Delta_{W}^{\frac{1}{2}}, S_{W} \psi=\psi\right\}, K(W) \text { closed } \\
J_{W} K(W)=K\left(W^{\prime}\right)=K(W)^{\prime}, \text { duality } \\
\overline{K(W)+i K(W)}=H, K(W) \cap i K(W)=0
\end{gathered}
$$

It is important to be aware that, unlike QM, we are dealing here with real (closed) subspaces $K$ of the complex one-particle Wigner representation space $H$. An alternative is to directly work with complex dense subspaces $K(W)+i K(W)$ (third line). Introducing the graph norm in terms of the positive operator $\Delta$, the dense complex subspace becomes a Hilbert space in its own right. The second and third line require some more explanation: the upper dash on regions denotes the causal disjoint (the opposite wedge), whereas the dash on real subspaces stands for the symplectic complement with respect to the symplectic form $\operatorname{Im}(\cdot, \cdot)$ on $H$.

The two properties in the third line are the defining relations of what is called the standardness property of a real subspace ${ }^{10}$; any standard $K$ space permits to define an abstract s-operator

$$
\begin{aligned}
S(\psi+i \varphi) & =\psi-i \varphi, \psi, \varphi \in K \\
S & =J \Delta^{\frac{1}{2}}
\end{aligned}
$$

\footnotetext{
${ }^{10}$ According to the Reeh-Schlieder [21] theorem a local algebra $\mathcal{A}(\mathcal{O})$ in QFT is in standard position with respect to the vacuum i.e. it acts on the vacuum in a cyclic and separating manner. The spatial standardness, which follows directly from Wigner representation theory, is just the one-particle projection of the Reeh-Schlieder property.
} 
whose polar decomposition (written in the second line) yields two modular objects, a unitary modular group $\Delta^{i t}$ and an antiunitary reflection $J$, which generally have however no geometric significance. The domain of the Tomita $S$-operator is the same as the domain of $\Delta^{\frac{1}{2}}$, namely the real sum of the $K$ space and its imaginary multiple. In our case this domain is determined solely in terms of Wigner group representation theory.

Coming from QFT, the complex $\operatorname{dom} S_{W}$ can be understood as the complex dense space which is provided by projecting the dense Reeh-Schlieder domain (obtained by applying fields smeared with W-supported test functions to the vacuum [21]) to the one-particle space, and the closed $K(W)$ space results from projecting only Hermitian operators. The modular localization approach provides a constructive access to QFT whithout quantization by avoiding any parallelism with the less fundamental classical field theory ${ }^{11}$. As will be demonstrated in section 4, this is quite easy in the absence of interactions, but leads to new concepts which pose novel problems in the presence of interactions (section 5 ). The $J$ transformed $K$-spaces (3) permit a more direct description in terms of symplectic complements

$$
J K=K^{\prime}:=\{\chi \mid \operatorname{Im}(\chi, \varphi)=0, \text { all } \varphi \in K\}
$$

It is easy to obtain a net of $\mathrm{K}$-spaces by $U(a, \Lambda)$-transforming the $\mathrm{K}$-space of a particular $W_{0}$. A bit more tricky is the construction of sharper localized subspaces via intersections

$$
K(\mathcal{O})=\bigcap_{W \supset \mathcal{O}} K(W)
$$

where $\mathcal{O}$ denotes a causally complete smaller region (e.g. non-compact spacelike cone, compact double cone). Intersection may not be standard, in fact they could even be zero, in which case the theory allows localization in $W$ (it always does), but not in $\mathcal{O}$. One can show that the intersection for non-compact spacelike cones $\mathcal{O}=\mathcal{C}$ is always standard for all positive energy representations [35].

Standardness for compact double cone regions $\mathcal{O}=\mathcal{D}$ leads to pointlike localized generating wave functions (wave-function-valued Schwartz distributions). This applies to $(m>0, s)$ and to massless finite helicity representations, whereas the Wigner massless infinite spin family with $\mathcal{K}(\mathcal{D})=0, K(\mathcal{C})$ standard, requires semiinfinite spacelike string generating wave functions. In the functorial relation between Wigner wave functions and quantum fields this leads to pointlike/stringlike localized generating free fields. The positive energy Wigner representations fall into 3 families: positive mass, zero mass finite helicity and zero mass infinite helicity. Only the third class, for which the two-dimensional Euclidean little group is faithfully represented, requires stringlike generating wave functions.

\footnotetext{
${ }^{11}$ In Jordan's terminology "without classical crutches" [65].
} 
Since all states in QFT carry a unitary representation of the Poincaré group which permits a (discrete or continuous) decomposition into irreducible components, this closes the issue of modular state-localization. Modular operator localization of free fields follows state-localization (section 4), however modular localization of interacting operator algebras requires a subtle refinement which accounts for new conceptual problems posed by interactions (section 5).

For the explicit construction of the pointline free fields of arbitrary mass and finite spin it is somewhat easier to follow Weinberg [34] and compute covariant intertwiners which map momentum space creation and annihilation operators into covariant fields. This is possible because in the absence of interactions modular localization and covariance are equivalent requirements and the differences between Wigner's "little group" and its representation accounts fully for the differences in localization between the three representation families.

Leaving out the string-localized infinite spin family, the result of the "covariantization" associates one unitary $(\mathrm{m}, \mathrm{s})$ Wigner representation with an infinite family of generating covariant spinorial wave functions $\Psi^{(A, \dot{B})}$ whose spinorial undotted/dotted spinorial indices are related to the physical spin s through the following inequalities ${ }^{12}$

$$
\begin{gathered}
|A-\dot{B}| \leq s \leq|A+\dot{B}|, m>0 \\
s=|A-\dot{B}|, m=0
\end{gathered}
$$

One notices that in the zero mass case the vector representation $(A=1 / 2, B=$ $1 / 2)$ for $\mathrm{s}=1$ and the $(A=1, B=1)$ for $\mathrm{s}=2$ are missing i.e. precisely those fields which correspond to the classic electromagnetic vectorpotential and for $\mathrm{s}=2$ to the metric tensor. These gaps in the massless case have important physical consequences.

Wigner's representation theory for positive energy representations of the Poincaré group combined with the calculation of intertwiners via covariantization represents a completely intrinsic quantum path to free fields. The passing from generating covariant wave function to covariant quantum fields only requires to reinterpret the momentum space wave functions $a^{*}(p)$ and their antiparticle counterpart $b(p)$ as canonical creation/annihilation operators

$$
\Psi^{(A, \dot{B})}(x)=\frac{1}{(2 \pi)^{3 / 2}} \int \frac{d^{3} p}{2 p_{0}}\left\{e^{i p x} u^{(A, \dot{B})}(p) \cdot a^{*}(p)+e^{-i p x} v^{(A, \dot{B})}(p) \cdot b(p)\right\}
$$

Here the dot stands for the summation over physical spin components and the dependence on the spinorial component of the $(A, \dot{B})$ representations (ranging over $2 A+1$ respectively $2 \dot{B}+1$ values) on the $\Psi$ and the $u, v$ intertwiners have been omitted. The covariantization leading to the intertwiners uses only group theory, it can be found in the first volume of Weinberg's well-known book [34].

\footnotetext{
${ }^{12}$ For convenience of notation our spinorial indices are half of the standard ones.
} 
There is a subtle consequence of modular localization which one encounters in the second ( $m=0, s \geq 1)$ representation class of massless finite helicity representations (the photon-graviton family). Whereas in the massive case the relation of the physical spin $s$ with the formal spin in the spinorial fields follows the angular momentum composition rules which leads to the spinorial restrictions (7) [34], the zero mass finite helicity family in the second line has a significantly reduced number of spinorial descriptions. Different from classical Maxwell theory, where pointlike vectorpotentials are perfectly acceptable (constrained) classical fields, their quantum counterparts do not appear in the covariantized Wigner's list (8).

The explanation of this dilemma, which also leads to its cure, is that the loss of pointlike quantum potentials is the result of a clash between the Hilbert space structure (positivity) and pointlike localization. The missing spinorial fields in (8), as compared to (7), reappear after relaxing the localization from pointlike to stringlike [37] [38]. Both kind of fields are singular limits of operators localized in causally closed regions; pointlike fields in case of double cone localized and semi-infinite stringlike fields in case of spacelike cone localized operators. Once one allows stringlike covariant fields i.e. $\Psi^{(A, \dot{B})}(x, e)$ localized on spacelike half-lines $x+\mathbb{R}_{+} e,(e=$ spacelike directional unit vector $)$, the full range of spinorial realizations $(7)$ is available. These generating free fields are covariant and "string-local"

$$
\begin{aligned}
U(\Lambda) \Psi^{(A, \dot{B})}(x, e) U^{*}(\Lambda) & =D^{(A, \dot{B})}\left(\Lambda^{-1}\right) \Psi^{(A, \dot{B})}(\Lambda x, \Lambda e) \\
{\left[\Psi^{(A, \dot{B})}(x, e), \Psi^{\left(A^{\prime}, \dot{B}^{\prime}\right)}\left(x^{\prime}, e^{\prime}\right]_{ \pm}\right.} & =0, x+\mathbb{R}_{+} e><x^{\prime}+\mathbb{R}_{+} e^{\prime}
\end{aligned}
$$

although the Wigner representation itself (and its functorially related local operator algebra, see next section) remains pointlike generated, since the field strengths suffice for its generation. But for other purposes the potentials are indispensable. (see below). The use of covariant string fields also facilitates the construction of finite "gauge bridges" (using the terminology of gauge theory) between two matter fields with opposite charges.

The third Wigner family, the infinite spin representations, resisted all attempts to understand their localization properties for a very long time. Its local generators have no analog in classical Lagrangian field theory and also did not appear in Weinberg's intertwiner formalism [34]. Only after the concept of modular localization was applied to Wigner's representation theoretical construction [35] [37], it became clear that this is a case of a string-like generated Wigner representation. The differences in localization properties can be traced back to the different representations of the Wigner "little groups". For $\mathrm{m}=0$ representations the little group is the two-dimensional Euclidean group, but only for the faithful $E(2)$ representation the non-compactness of the group makes itself felt in the necessity to introduce stringlike generators. The interwiners do not have spinorial indices, instead they depend on a string direction $e$ and the dot in (9) stands for an infinite sum reflecting the infinite dimensional nature of the $E(2)$ representation space. 
The conventional description of vectorpotentials, which one obtains from quantization of their classical counterpart, maintains the pointlike formalisms at the expense of Hilbert space structure. The application of the BRST formalism gives the physically correct results only for gauge invariant quantities, which are automatically pointlike localized [40]. But the physical electric charge-carrying operators are known to allow no localization which is sharper then an arbitrary thin spacelike cone with stringlike generating fields which remain outside the BRST formalism. The use of the string-localized vectorpotentials exposes the origin of their string-like localization by coupling the quantum matter to stringlike vectorpotentials. The expected result is that their stringlike localization is exported to the matter field, which in zero order perturbation was pointlike localized. Whereas the vectorpotential continues to lead to pointlike field strengths, there is no linear operation which undoes the string localization of the charge-carrying field.

Even in the case of free fields the use of stringlike vectorpotentials protects against incorrect application of the gauge formalism. A well-known illustrations is the Aharonov-Bohm effect in $\mathrm{QFT}^{13}$. It is not necessary to use vectorpotentials, but if one decides to use them it is important to work with the stringlocalized potential since the pointlike indefinite metric potential (the Feynman gauge) gives a wrong answer [41][40].

Stringlike massless higher spin "potentials" have a better short distance behavior than their associated field strength (whose short distance dimension increases with $s$ ); in fact in all cases one finds potentials with $d_{s d}=1$ which is the smallest dimension allowed by Hilbert space positivity and also the largest for satisfying the power counting renormalization requirement in up to quadrilinear polynomial couplings. As a result there are candidates for renormalizable interactions for any spin. Of course renormalization theory is more than powercounting; one also has to show that an extension of the Epstein-Glaser iterative implementation of causal commutativity can be implemented for stringlike fields.

Stringlike localized covariant fields can also be constructed in massive higher spin theories. In this case the pointlike potentials exist, but their dimensions increases with $s$. The string-like description and the BRST formalism both use massive potentials with $d_{s d}=1$. Even the relation to the pointlike physical Proca potential is formally similar [42][43]

$$
\begin{aligned}
& A_{\mu}(x, e)=A_{\mu}^{P}(x)+\partial_{\mu} \phi(x, e), A_{\mu}^{B R S T}(x)=A_{\mu}^{P}(x)+\partial_{\mu} \phi^{S}(x) \\
& \left\langle A_{\mu}(x, e) A_{v}\left(x^{\prime}, e^{\prime}\right)\right\rangle=\frac{1}{(2 \pi)^{3}} \int \frac{d^{3} p}{2 p_{0}} e^{-i p\left(x-x^{\prime}\right)}\left\{-g_{\mu \nu}-\right. \\
& \left.-\frac{p_{\nu} p_{\nu}\left(e, e^{\prime}\right)}{(p e)_{-i \varepsilon}\left(p e^{\prime}\right)_{i \varepsilon}}+\frac{p_{\mu} e_{\nu}}{(p e)_{-i \varepsilon}}+\frac{p_{\mu} e_{\nu}^{\prime}}{\left(p e^{\prime}\right)_{i \varepsilon}}\right\}
\end{aligned}
$$

where the $\varepsilon$-prescription refers to the way in which the real boundary in $e$ has to be approached. The difference in (11) is that the scalar Stückelberg field $\phi^{S}(x)$ has the opposite metric (it is related to $A_{\mu}^{B R S T}(x)$ via the s-operation of

\footnotetext{
${ }^{13}$ The standard A-B effect refers to QM in an external magnetic field.
} 
the BRST formalism $s A_{\mu}^{B R S T}=\partial_{\mu} u, s \phi^{S}=u$ ). The use of the total derivative $\partial_{e}:=\sum \frac{\partial}{\partial e^{\alpha}} d e^{\alpha}$ leads to an even stronger formal connection

$$
\begin{gathered}
\partial_{e} A_{\mu}(x, e)=\partial_{\mu} v(x), \partial_{e} \phi(x, e)=v(x) \\
s A_{\mu}^{B R S T}(x)=\partial_{\mu} u(x), s \phi^{S}(x)=u(x)
\end{gathered}
$$

where $v$, the counterpart of $u$, is pointlike [43]. This stringlike description is reminiscent of Mandelstam's attempt [44] to avoid indefinite metric by expressing the dynamics in terms of field strength only. Indeed a stringlike potential is uniquely determined by the field strength and a spacelike direction $e$; but the introduction of a separate operator $A_{\mu}(x, e)$ which fluctuates in both $x$ and $e$ is preferable, because the improvement of the short distance $x$-fluctuation which is crucial for renormalization, and its prize in terms of the appearance of infrared fluctuations, is placed into evidence.

The more surprising consequences of modular localization are certainly those which appear in the title of this paper; they will be presented in section 5 .

\section{Some expected consequences}

The stringlike reformulation of gauge theories leads to a setting which has some formal similarities with the BRST formalism ${ }^{14}$ without suffering from its limitations. Its use for massive vectormesons relates their string-localized description, which has the mild short distance behavior needed for renormalizability, with the standard physical description in terms of pointlike Proca field, similar to the relation between the pointlike indefinite metric BRST vectorpotential with the Proca field (11). There are reasons to believe that such a relation has its multiplicative counterpart in a relation between a stringlike interacting matter field (unavoidable as the result of its coupling with the string-localized potential) and a multiplicatively corresponding pointlike matter field which together with the pointlike vectormeson leads to a better description (after the issue of renormalization has been settled). Both set of fields belong to the same theory, they are relatively local generating fields acting in the same Hilbert space. The pointlike fields permit to present the result in the standard way, but in contradistinction to interactions between fields with $s<1$, they are not suitable for staying within the power-counting limit required by renormalization. In contrast to the BRST description one does not need Krein spaces; the role of the short distance-improving indefinite metric $A_{\mu}^{B R S T}$ is now taken over by the stringlike vectorpotential which, apart from being string-localized, is expected to belong to the same QFT as the Proca field.

The conceptual advantage of the stringlike formulation emerges in a much stronger form in the zero mass limit. The Proca potential cannot have a zero mass limit since a physical (Hilbert space) pointlike zero mass vectorpotential does not exist. In fact there are two different BRST settings [45]; one for

\footnotetext{
${ }^{14}$ Concerning the application of the BRST formalism to massive vectormeson we follow Scharf's book [45].
} 
massive vectormesons, in which case the BRST formalism is only used on the level of the free asymptotic fields, and the other for the massless gauge theories, where the BRST s-operation has a perturbative dependence of the coupling strength. In the stringlike formulation everything is under one conceptual roof, the pointlike potentials are only lost in the zero mass limit. Computation with stringlike fields should be done for massive vectormesons; one hopes that the connection of string-localization and infrared properties is more amenable than in the pointlike case.

For the matter fields, the main change between the stringlike matter fields and its would-be multiplicative related pointlike counterpart is expected to occur in the long-distance regime, whereas for short distances there should be essentially no change. For nonabelian zero mass models one naturally expects that the linear field strength remains stringlike and only appropriate composites maintain their pointlike localization i.e. the logic of classical gauge invariance is replaced by the identification of a pointlike generated observable substrate embedded in a string-generated QFT.

Approaching QED from the massive string-localized side has the advantage that physical charged fields (infraparticle fields), which have been known for a long time to acquire a non-compact localization [21], are now part of the perturbative formalism. Whereas in the existing formulation they only appear as a computational device in a prescription for computing on-shell photon inclusive scattering cross sections, their expected new role is to represent electric charge-carrying physical "infraparticle" fields with admit (off-shell) correlation function. The largest gain of insight from string-localization is expected to come from approaching Yang-Mills theories via massive self-interacting vectormesons. Whereas the physical origin of infrared singularities of the unphysical pointlike matter fields in the BRST setting remains unexplained (or is blamed on a not yet understood nonperturbative regime), the stringlike formulation may reveal a different story.

The string-localization plays a pivotal role for the understanding of the offshell infrared divergencies of self-interacting massive vectormesons in the zero mass limit. In the nonabelian case only color-neutral composites of the field strengths are expected to maintain their pointlike localization in the massless limit. It is perfectly conceivable that gluon strings exist in the Hilbert space but that the expectation of the energy-momentum operator in a state created from the vacuum by a gluon operator leads to a diverging global energy.

This could have interesting consequences for the asymptotic freedom issue and for the confinement idea. A beta-function computed in the dimensional regularization formalism without the Callan-Symanzik equation from where it originates remains incomplete. One needs to represent the infrared-divergent massless Yang-Mills theory as the massless limit of a perturbatively accessible massive theory ${ }^{15}$ in order to derive a C-S equation. The existing derivation of asymptotic freedom is tantamount to a consistency argument: the inverse sign

\footnotetext{
${ }^{15}$ The prototype illustration is the derivation of the C-S equation of the massive Thirring model. In this case the vanishing of beta to all orders insures the existence of the massless limit as a conformal QFT [46].
} 
of the beta function is consistent with the expected perturbative short distance behavior of a nonexisting renormalized perturbation theory.

The reformulation of Y-M interaction in terms of stringlike vectorpotentials permits to question this scenario; massive stringlike selfinteracting vectormesons may have a massless limit, a situation which cannot be achieved in the pointlike BRST formalism. Assuming that Callan-Symanzik equations also can be established in the stringlike setting, such a step would combine massive $s=1$ fields and their massless limit under one roof, just as it is well-known in case of interactions with $s<1$ for which this limit for pointlike fields. Such a scenario, if it can be made to work, could change the whole perspective on QFT and its use in particle physics. String-localized potentials with their string-improved short distance dimension $d_{s d}=1$ are potential candidates for renormalizable interactions between all $(m, s \geq 1)$ quantum fields.

The second area which could suffer significant modifications is the issue around the Higgs boson. From a "Schwingerian" point of view what remains of the complex matter field in the screened "phase" of scalar QED (which is a three-parametric renormalizable QFT in terms of $m_{s c}, g_{s c}, e$ ) is a real scalar matter field, after its remaining real partner participated in the conversion of the photon into a massive vectormeson. The screened phase of QED is one in which the Maxwell charge (related to the identically conserved current in $\partial^{\mu} F_{\mu \nu}=j_{\nu}$ ) is "screened" i.e. the integral over $j_{0}$ vanishes. Schwinger's screening idea ${ }^{16}$ is a QFT extension of Debye screening in QM, except that in QFT this is more radical (change of particle spectrum). This idea was later backed up by a theorem due to Swieca [47][48] which states that this situation permits precisely two alternative realizations: either the charge is $\neq 0$, in which case the massshell is converted into the milder cut singularity of infraparticle, or there is a mass gap which leads to a zero charge (the screening phase).

Its discoverers Higgs [49] and [50][51] presented the result as a two step process in which a spontaneous symmetry breaking is followed by a transfer of the massless Goldstone boson to the vectorpotential which, as a result of gaining an additional degree of freedom, becomes a massive vectormeson. This idea was helpful from a computational viewpoint (since the perturbative Goldstone spontaneous symmetry breaking mechanism was well-known at the time of Higgs). The result is the same as that computed from Schwinger-Higgs screening except that there is no intermediate spontaneous symmetry breaking. Unfortunately the terminology "broken gauge invariance" led to a rather widespread incorrect understanding of the Higgs mechanism. There is no symmetry breaking, unless one wants to consider the appearance of odd terms in the self-interaction of the remaining screened (real) scalar field as a spontaneous $\mathbb{Z}_{2}$ symmetry breaking.

The renormalization theory of massive vectormesons appeared first in the form of massive QED [52]. Some loose ends of that treatment concerning the passing from the indefinite metric description to the unitary gauge were over-

\footnotetext{
${ }^{16}$ Schwinger's attempt to find a perturbative realization in spinor QED failed and he instead used two-dimensional QED which became known as the "Schwinger model". He was apparently not aware that by replacing spinors by complex scalar fields he could have had a perturbative realization of his idea [48].
} 
come in the BRST formalism. In recent work [53][54] which extends Scharf's [45] pathbreaking work ${ }^{17}$ on the BRST setting of massive vectormesons, the absence of any symmetry breaking is emphasized against the mainstream view. To the extend that the mainstream pays attention to this work, also the interest in Schwinger-Higgs screening and the screening theorem by Swieca (which had a foundational impact on the development of $\mathrm{LQP}^{18}$ but never made it into the mainstream) may receive the attention it deserves.

The conclusion of absence of symmetry breaking in the massive BRST formulation is based on the observation that its typical formal indicators do not appear in this setting [45]. The intrinsic (independent of the computational method) argument for the presence of the Schwinger-Higgs screening is however the vanishing of the Maxwell-charge associated with the identically conserved (massive) Maxwell current. In contrast the conserved current in the Goldstone spontaneous symmetry breaking model diverges as the result of its coupling to the massless Goldstone boson. The screening picture may not be helpful in perturbative computations, but it quite easy to verify that the mass gap in the BRST leads to the screened Maxwell charge.

The most exiting application of the stringlike formalism is certainly its use for the clarification of the Higgs issue: are self-interactions of vector-mesons only possible in the presence of a Higgs particle? The BRST formalism leads to an affirmative answer [45] but it would be better to understand this from the localization principle instead of leaving it to the consistency of the indefinite matric BRST formalism. If this result is not confirmed, all the hype about the world of QFT breaking down without a Higgs particle was irrelevant and the presentation of LHC results with two competing theoretical results would liberate particle physics from its self-generated religious-ideological freight. If on the other hand the stringlike setting confirms the necessity of the Higgs, it will present the best starting point to find out whether the presence of low spin satellites of (self)interacting higher spin massive particles is a general consequence of modular localization or whether this is a peculiarity of selfinteracting massive vectormesons.

Apart from some mathematical problems as adjusting the Epstein-Glaser renormalization framework to the needs of string-localization [42], the calculations for most of the mentioned problems are still in progress [55]. Stringlike perturbation requires new concepts and is more involved; the pointlike massive fields in terms of which one hopes to express the result of massive vectormeson interactions are not the same fields as those which are used to stay below the power-counting limit.

As one does not have to understand details about causal localization for handling the renormalization theory of pointlike fields, one expects to find rules for perturbing couplings involving stringlike fields which avoid the intricacies of

\footnotetext{
${ }^{17}$ Besides his formulation of operator gauge invariance Scharf uses only the intrinsic logic of the BRST formalism.

${ }^{18}$ It led Buchholz and Fredenhagen to discover the connection of spectral gaps with the stringlike generating property of superselected charge-carrying fields (as the only possibility which replaces the pointlike localization in case of non-existence of pointlike generators) [21].
} 
modular localization and lead to a simple description between stringlike fields needed for renormalization and their pointlike counterparts. Since in [37] the idea of their use originated from the desire of a more detailed understanding of the work on modular localization [35] in the setting of Wigner's representation theory, this connection with problems of "real particle physics" alluded to in this section may also raise the readers interest in the more abstract presentation of modular localization in the following sections.

It is somewhat harder to speculate whether there could be some physical use for the "kinematical" strings which are the generator of the string-localized Wigner infinite spin representation. It is hard to imagine that any kind of compactly localized counter can detect them or that they can be produced from local interactions of ordinary particles. Weinberg [34] dismissed the infinite spin representations on the ground that "nature does not use them", but it is questionable whether in times of dark matter one can uphold this dismissal.

A yet different kind of spacelike strings arises in $d=1+2$ Wigner representations with anomalous spin [39]. The modular localization approach preempts the spin-statistics connection already in the one-particle setting, namely if $\mathrm{s}$ is the spin of the particle (which in $\mathrm{d}=1+2$ may take on any real value) then one finds for the connection of the symplectic complement with the causal complement the generalized duality relation

$$
Z K\left(\mathcal{O}^{\prime}\right)=K(\mathcal{O})^{\prime}
$$

where the square of the twist operator $Z=e^{\pi i s}$ is easily seen (by the connection of Wigner representation theory with the two-point function) to lead to the statistics phase $=Z^{2}[39]$. Here the strings have a more virtual existence, they serve to keep track of localization of plektons when the representation of the universal covering group is activated by anomalous spin but differential geometry does not provide a spacetime covering. Different from all previous cases these representations have no functorially associated free fields; surprisingly the QFT of anyons and plektons (nonabelian representations of the braid group) do not even permit integrable models [56].

\section{Algebraic aspects of modular theory}

A net of real subspaces $K(\mathcal{O}) \subset H_{1}$ for a finite spin (helicity) Wigner representation can be "second quantized" ${ }^{19}$ via the CCR (Weyl) respectively the CAR quantization functor; in this way one obtains a covariant $\mathcal{O}$-indexed net of von Neumann algebras $\mathcal{A}(\mathcal{O})$ acting on the bosonic or fermionic Fock space $H=$ Fock $\left(H_{1}\right)$ built over the one-particle Wigner space $H_{1}$. For integer spin/helicity values the modular localization in Wigner space implies the identification of the symplectic complement with the geometric complement in the sense of relativistic causality, i.e. $K(\mathcal{O})^{\prime}=K\left(\mathcal{O}^{\prime}\right)$ (spatial Haag duality in $H_{1}$ ). The Weyl

\footnotetext{
${ }^{19}$ The terminology "second quantization" is a misdemeanor since one is dealing with a rigorously defined functor within QT which has little in common with the artful use of that parallellism to classical theory called "quantization".
} 
functor takes this spatial version of Haag duality into its algebraic counterpart, whereupon the symplectic complement passes to the von Neumann commutant subalgebra.

One proceeds as follows: for each Wigner wave function $\varphi \in K(\mathcal{O}) \in H_{1}$ the associated (unitary) Weyl operator is defined as

$$
\begin{gathered}
W \operatorname{eyl}(\varphi):=\operatorname{expi}\left\{a^{*}(\varphi)+a(\varphi)\right\} \in B(H) \\
\mathcal{A}(\mathcal{O}):=\operatorname{alg}\{W \operatorname{eyl}(\varphi) \mid \varphi \in K(\mathcal{O})\}^{\prime \prime}, \quad \mathcal{A}(\mathcal{O})^{\prime}=\mathcal{A}\left(\mathcal{O}^{\prime}\right)
\end{gathered}
$$

where $a^{*}(\varphi)$ and $a(\varphi)$ are the usual Fock space creation and annihilation operators of a Wigner particle with wave function $\varphi$. This is a functorial relation between localization subspaces of the one-particle space and localized subalgebras. Defining the algebra in terms of the double commutant converts it into a von Neumann algebra i.e. a weakly closed operator algebra.

This functorial relation between real subspaces and von Neumann algebras via the Weyl functor preserves the causal localization and commutes with the improvement of localization through intersections $\cap$ according to

$$
K(\mathcal{O})=\cap_{W \supset O} K(W), \mathcal{A}(\mathcal{O})=\cap_{W \supset O} \mathcal{A}(W)
$$

The functorial relation can be conveniently expressed in the commuting diagram

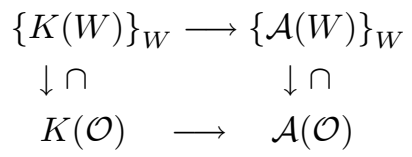

Here the vertical arrows denote the tightening of localization by intersections whereas the horizontal ones stand for the action of the Weyl functor. This commuting diagram expresses the functorial relation between particles and fields in the absence of interactions and represents a straightforward functorial extension of Wigner's representation theory ${ }^{20}$. In the interacting case this functorial connection is lost and the particle-field relations becomes significantly more subtle. Its conceptual/mathematical complexity in the case of non-integrable interactions is to blame for the 80 years lasting lack of progress in proving even the mathematical existence of a model behind its Lagrangian description, not to mention the problem of controlled approximations. A new attempt concerning this age-old problem which is based on the distinguished role of wedge-localized subalgebras will be presented in the next section.

The case of half-integer spin representations is analogous [36], apart from the fact that there is a mismatch between the causal and symplectic complements which must be taken care of by a twist operator $Z$; as a result one has to use the CAR functor instead of the Weyl functor. In $d=1+2$ one encounters an exception; the Bargman-Wigner representation theory permits anomalous spin which turns out to be connected with braid group statistics. As already

\footnotetext{
${ }^{20}$ Here we consider modular localization as part of Wigner's theory because the modular localization is constructed within positive energy representations of the Poincaré group [35].
} 
mentioned, this is the only known case for which (for $s \neq$ semiinteger) there is no functorial relation between localized subspaces and localized von Neumann subalgebras.

In case of the large family of irreducible zero mass "infinite spin" representations, for which the lightlike "little group" is faithfully represented, the functorial relation leads to string-localized generating fields which generate the Fock space and the non-compact localized subalgebra acting in it. There is an outline of an argument which excludes the existence of compact-localized (generated by pointlike composites) subalgebras [37], but unfortunately it is not conclusive.

A discrete basis of local covariant field coordinatizations is defined by Wick composites of the free fields. The case which deviates furthest from classical behavior is the pure stringlike infinite spin representation for which the class of relative string-localized fields form a continuous family of composites. Its non-classical aspects, in particular the absence of a Lagrangian, is the reason why the spacetime description in terms of semiinfinite string fields has been discovered only more than 60 years after it appeared in Wigners classification [35][37].

Using the standard notation $\Gamma$ for the second quantization functor which maps real localized (one-particle) subspaces into localized von Neumann algebras, and extending this functor in a natural way to include the images $\Gamma($.$) of$ the $K(\mathcal{O})$-associated modular objects (denoting the spatial one-particle objects in section 2 now as $s, j, \delta$ ) one arrives at a special case of the Tomita Takesaki modular theory for the interaction-free standard pair $(\mathcal{A}(\mathcal{O}), \Omega)^{21}$

$$
\begin{aligned}
& H_{\text {Fock }}=\Gamma\left(H_{1}\right)=e^{H_{1}}, \quad\left(e^{h}, e^{k}\right)=e^{(h, k)} \\
& \Delta:=\Gamma(\delta), J:=\Gamma(j), S:=\Gamma(s) \\
& S A \Omega=A^{*} \Omega, A \in \mathcal{A}(\mathcal{O}), S=J \Delta^{\frac{1}{2}}
\end{aligned}
$$

The Tomita-Takesaki theorem is about the action of the two modular objects $\Delta^{i t}$ and $J$ on the algebra

$$
\begin{aligned}
\sigma_{t}(\mathcal{A}(\mathcal{O})) & \equiv \Delta^{i t} \mathcal{A}(\mathcal{O}) \Delta^{-i t}=\mathcal{A}(\mathcal{O}) \\
J \mathcal{A}(\mathcal{O}) J & =\mathcal{A}(\mathcal{O})^{\prime}=\mathcal{A}\left(\mathcal{O}^{\prime}\right)
\end{aligned}
$$

in words: the reflection $J$ maps an algebra (in standard position) into its von Neumann commutant and the unitary group $\Delta^{i t}$ defines an one-parametric automorphism-group $\sigma_{t}$ of the algebra. In this form (but without the last geometric statement involving the geometrical causal complement $\mathcal{O}^{\prime}$ ) the theorem hold in complete mathematical generality for "standard pairs" $(\mathcal{A}, \Omega)$. The free fields and their Wick composites are "coordinatizing" singular generators of this $\mathcal{O}$-indexed net of operator algebras in the sense that the smeared fields $A(f)$ with suppf $\subset \mathcal{O}$ are (unbounded operators) affiliated with $\mathcal{A}(\mathcal{O})$ and generate $\mathcal{A}(\mathcal{O})$ in an appropriate mathematical sense.

\footnotetext{
${ }^{21}$ The functor second quantization functor $\Gamma$ preserves the standardness i.e. maps the spatial one-particle standardness into its algebraic counterpart.
} 
Within the classifications of von Neumann algebras these local algebras are of a very different type as their global counterpart. The latter is of the same type as quantum mechanical algebras, namely an algebra of all bounded operators on a Hilbert space $B(H)$. The local subalgebras are however isomorphic to the aforementioned monad [21]. More important than understanding its position within Connes type classification for our purpose is to characterize a monad by its use in physics. This is facilitated by the fact that there are only two types which one needs for the formulation of continuous ${ }^{22}$ quantum physics: type $I_{\infty}$ (or $B(H)$, the algebra of all bounded operators on a Hilbert space) in QM, and monads as localized subalgebras in QFT [57], where only the global algebra is a $B(H)$.

The relevance of the T-T modular theory for interacting QFT is based on the standardness of $(\mathcal{A}(\mathcal{O}), \Omega)$ (more generally for all finite energy states) which is a consequence of the Reeh-Schlieder theorem [21]. The definition of the Tomita involution $S$ through its action on the dense set of states (guaranteed by the standardness of $\mathcal{A}$ ) as $S A \Omega=A^{*} \Omega$, and the action of the two modular objects $\Delta, J(15)$ is part of the general setting of the modular Tomita-Takesaki theory of abstract operator algebras in "standard position"; standardness is the mathematical terminology for the physicist's Reeh-Schlieder property i.e. the property of that the action of a localized subalgebra on the vacuum vector ${ }^{23}$ $\Omega \in H$ is cyclic $\overline{\mathcal{A}(\mathcal{O}) \Omega}=H$ and $\mathcal{A}(\mathcal{O})$ contains no "annihilators" of $\Omega$.

The important property which renders this useful as a new constructive tool in the presence of interactions, is that for $(\mathcal{A}(W), \Omega)$ the antiunitary involution $J$ depends on the interaction, whereas $\Delta^{i t}$ continues to be uniquely fixed by the representation of the Poincaré group i.e. by the particle content. In fact it has been known since Jost's seminal work on TCP [58] (including the TCP covariance of the S-matrix) that the interacting TCP operator is related to its free (incoming) counterpart through the S-matrix; the $J$, which represents the reflection on the edge of the wedge, only differes from TCP by a $\pi$-rotation. Rewritten in terms of the reflection $J$ on the edge of a wedge this reads as

$$
J=J_{0} S_{\text {scat }}
$$

In this form it attributes the role of a relative modular invariant (between the interacting and free wedge-localized algebra) to the S-matrix and as a result became a constructive tool of QFT [14].

It is precisely this "semilocal" property of $S_{\text {scat }}$ in connection with wedgelocalization which opened the way for the inverse scattering construction within the setting of the bootstrap-formfactor project.

The physically relevant facts emerging from modular theory in the general setting can be condensed into the following statements:

\footnotetext{
${ }^{22}$ For discete combinatorial algebraic structures, as one encounters them in lattice theories, also type $\mathrm{II}_{1}$ enters, see remarks in last section.

${ }^{23}$ In QFT any finite energy vector (which of course includes the vacuum) has this property, as well as any nondegenerated KMS state.
} 
- The domain of the unbounded operators $S(\mathcal{O})$ is fixed in terms of intersections of the wedge localized algebras $\mathcal{A}(\mathcal{O})=\cap_{W \supset \mathcal{O}} \mathcal{A}(W)$. The domains associated to $S(W)$ are determined by the representation of the Poincare group (and hence by the particle content alone). These dense domains change with $\mathcal{O}$ i.e. the dense set of localized states has a bundle structure.

- The complex domains Dom $S(\mathcal{O})=K(\mathcal{O})+i K(\mathcal{O})$ in $H_{\text {Fock }}$ decompose into real subspaces $K(\mathcal{O})=\overline{\mathcal{A}(\mathcal{O})^{\text {sa }} \Omega}$. This decomposition contains dynamical information which in case $\mathcal{O}=W$ includes the $S_{\text {scat }}$-matrix (17). In the next section arguments will be presented which suggests that with the help of a new emulation formalism, which extends Wigner's representation approach to the presence of interactions, the $S_{\text {scat-matrix under }}$ appropriate conditions may fix $\mathcal{A}(W)$ uniquely.

- The restriction of the vacuum state to a local operator algebra $\mathcal{A}(\mathcal{O})$ leads to a KMS relation at inverse modular temperature $\beta=1$

$$
\langle A B\rangle=\left\langle B e^{-H_{\text {mod }}} A\right\rangle, e^{-i t H_{\text {mod }}}:=\Delta^{i t}
$$

This localization-caused thermal behavior is accompanied by an area proportional localization-entropy (section1). It has a variety of important physical consequences.

Modular localization is intimately connected to the holistic aspect ${ }^{24}$ of QFT which places this theory into a sharp contrast with QM (even in relativistic QM in the form of the direct particle theory (DPI) [60]. A one-dimensional quantum mechanical chain or string of oscillators can be embedded into a space of arbitrary dimensions since quantum mechanical (Born) localization is not an intrinsic aspect of QT. On the other hand an embedding of a lower-dimensional into a higher dimensional QFT is not possible; or to phrase it the other way around: the restriction of a QFT to a lower dimensional submanifold "remembers" (as a result of its holistic nature) that it is the restriction of a more complete theory. In particular it is not possible to embed a one-dimensional chiral theory into a higher dimensional QFT; a fact which is overlooked in the incorrect picture of an embedding of a chiral theory into its inner symmetry space ("target space") used in string theory (see the last section for more remarks).

The holistic intrinsic nature of QFT presents itself most forcefully in the possibility of characterizing a quantum field theory by the positioning of a finite number of copies of an abstract monad in a shared Hilbert space. A "modular inclusion" of one monad into another defines a chiral QFT, for a 3-dimensional theory one needs 4 modular-positioned monads, and placing 7 monads into a specific modular position leads to $\mathrm{d}=1+3$ QFTs [61][60]. The positioning in Hilbert space determines not only the algebraic substrate (the kind of quantum matter) of a QFT, but also reveals its Minkowski spacetime localization properties and the action of the Poincaré group on it. The interpretation of a modular

\footnotetext{
${ }^{24}$ For emphasising the importance of this property for the issue of the cosmological constant we refer to the paper: "Quantum Field Theory Is Not Merely Quantum Mechanics Applied to Low Energy Effective Degrees of Freedom" by Hollands and Wald [59].
} 
inclusion of two monads is context-dependent; if one does not place additional monads into the same Hilbert space, it defines a chiral theory on which the Möbius group acts; a larger number of appropriately placed monads leads to higher dimensional local nets of operator algebras. It is this intrinsic relation of the abstract algebraic modular positioning of a finite number of monads in a Hilbert space to the concrete localization of quantum matter in spacetime (GPS positioning for LQP) that provides the strongest illustration of "holistic"; there is no other theory of quantum matter which is "relational" in this extreme way. As mentioned it forbids embedding of a lower into a higher dimensional QFT and places severe restrictions on "dimensional reduction" in QFT. Quantization is not a boundless game in which classical manipulations can be interchanged with quantization; the holistic nature of QFT shows its limitation and at the same time opens new perspectives. The problem is that one cannot see these limitations on the level of Lagrangian quantization; they would become visible if one tries to "curl up" extra dimensions in explicitly computed correlation functions by a mathematically controlled operation on their correlations function which maintains the holistic nature of $\mathrm{QFT}^{25}$ instead of manipulationing Lagrangians, which is the way the Kaluza-Klein dimensional reduction works in classical field theory.

It is interesting to briefly look at the difficulties which our QFT ancestors encountered with these holistic aspects. From the time of the "Einstein-Jordan conundrum" [62] through Jordan's subsequent discovery of QFT, Heisenberg's discovery of vacuum polarization, Unruh's Gedankenexperiment and Hawking's radiation up to the problem of the origin of the cosmological constant, in all those cases the holistic nature of QFT asserts itself.

\section{5 "Emulation" as an extension of Wigner's rep- resentation theoretical setting}

The bootstrap-formfactor program [11] was based on the assumption that the multiparticle components of a localized excitation of the vacuum $A|0\rangle, A \in$ $\mathcal{A}(\mathcal{O})$ in theories in $\mathrm{d}=1+1$ with an "integrable" S-matrix (purely elastic 2particle scattering ${ }^{26}$ ) written in terms of rapidity variables are meromorphic functions which, besides the degenerate represesentation of the permutation group from particle statistics, possess a different nontrivial representation of this group under analytic exchanges (through analytic continuations) of rapidities.

This led the above authors to a change in notation in which the statistics degeneracy is removed by encoding it into the left to right decreasing order of

\footnotetext{
${ }^{25}$ If the model has sufficient analyticity properties which allow real/imaginary time Wickrotations, one can "curl up" a time component by taking the high temperature limit in a KMS state und create a new time direction by Wick rotation. But this is not what the proponents of Kaluza-Klein reductions in QFT have in mind.

${ }^{26}$ The elastic two-particle amplitude in $\mathrm{d}=1+1$ is the only scattering amplitude which cannot be distinguished from the identity contribution by cluster factorization (equality of the product of two particle plane wave inner products with the energy-momentum delta function), by which factorizing models indicate their kinematical proximity to free fields.
} 
the numerical values of rapidities

$$
\left\langle 0|A| \theta_{1}, \theta_{2}, . . \theta_{k}\right\rangle, \theta_{1}>\theta_{2}>. .>\theta_{k}
$$

so that other orderings can be used to represent the result of analytic changes of ordering. The basic analytic change, the analytic transposition between two adjacent $\theta$, is defined in terms of a crossing symmetric unitary two-particle S-matrix ${ }^{27}$. From this analytic transposition one then constructs an analytic representation of the permutation group. In contrast to the degeneracy of the statistics representation which can be "dumped" into the ordering prescription, this analytic representation is encoded into the changes of orders of rapidities in the vacuum formfactor (18).

Together with the crossing property which connects the multi-particle components of local excitations of the vacuum $A|0\rangle$ with particle formfactors between arbitrary multi-particle states together with some general wisdom from LSZ scattering theory, this was basis of the bootstrap-formfactor construction of a QFT. Within these rules the so called inverse scattering problem admits a unique solution. In contrast to the perturbative approach based on Lagrangian or functional quantization, the only name for most of the models constructed in this way comes from their scattering function; a few S-matrices (the most prominent is the Sine-Gordon model) permit a perturbative relation with a Lagrangian from which these models inherit their classical name.

This analytic representation of the permutation group called for an algebraization in terms of a new kind of noncommuting particle operators. This was formally achieved in [12] and the associated algebra became known as the Zamododchikov-Faddeev (Z-F) algebra (after Faddeev added a missing cnumber term). For a long time the physical interpretation of these operators remained a mystery. Since the central property of QFT is causal localizability, it was natural to look for a relation of these generating operators to the localization properties of QFT. The deviation of the Z-F commutation relations from those of the standard creation/annihilation operators exclude pointlike localization of their Fourier transforms.

As explained in the first two sections, a particular well-suited formulation of QFT for unravelling the spacetime significance of operators is Haag's "local quantum physics" (LQP) which places a net of localized observable algebras and their "charged" representation sectors (from which together with the observables one can construct a charge-carrying "field-net") into the center stage and assigns to pointlike covariant fields the role of generators of localized algebras without attaching a preferential status to a particular field (apart from conserved currents which arise from the local implementation of global symmetries). An important step in the development of an intrinsic algebraic description was the aforementioned observation by Bisognano and Wichmann [31] (and its subsequent application to the Unruh effect and Hawking's black thermal radiation by Sewell [63]) that localization within a spacetime wedge has a deep

\footnotetext{
${ }^{27}$ These elastic S-matrices were obtained from the classification of solutions of a "bootstrap" project for scattering functions (solutions of the Yang-Baxter equations in case of matrix valued scattering functions) [11].
} 
relation to the Tomita-Takesaki modular theory of operator algebras including its thermal (KMS) aspects.

The application of these ideas to the bootstrap-formfactor project resulted [64] in the identification of the Fourier transforms of the Z-F operators with generators of wedge algebras for integrable models. As free fields, their one-time application to the vacuum state creates a one-particle state, but different from the former, the presence of infinite vacuum polarization clouds in interacting QFTs can in general not be avoided in their iterative application to the vacuum. The Z-F operators turned out to be special realizations of polarization-freegenerators (PFGs) in which the relation between particles and fields resembled those in free field models.

A subsequent more foundational study based on "modular localization" [18] revealed that there exist two types of PFGs, temperate ones, whose domains are translation invariant and whose particlelike Fourier transforms fulfill Z-F algebra commutation relations), and nontemperate PFGs which exist as operator-valued distributions only on wedge-localized test functions ${ }^{28}$ whose analytic on-shell restriction are only dense in the $L^{2}$ integrable space of all wave functions. It turns out that temperate PFGs only [17] exist in $\mathrm{d}=1+1$ and correspond to the family of integrable models (factorizing models) of the bootstrap-formfactor program [18]. Within this restricted set of temperate wedge-generators, the so-called inverse problem $\left(S_{\text {scat }} \rightarrow Q F T\right)$ has a unique solution in that the (necessarily) elastic $S_{\text {scat }}$ determines precisely one net of operator algebras (or a Borchers equivalence class of local fields [21]). The simpler particle-field connection, which comes with temperateness and accounts for all models which are integrable in the sense of the bootstrap-formfactor program, led to view temperateness of PFG generators of wedge-localized algebras as the foundational characterization of integrability of the associated QFT.

This new understanding of integrability in terms of localization reached its present final touch in the work of Lechner [16][4] who, by solving the existence problem (the existence of a nontrivial net of compact localized operators by intersecting wedge algebras) for many integrable models, contributed an important constructive step to the almost 90 year search for the mathematical control of QFT.

The resulting setting of integrable QFTs should be viewed as the generalization of Wigner's representation theoretical approach for the classification of one-particle spaces and a functorial construction of free field algebraic nets in the presence of interactions (see previous section). Wigner's motivation was that local quantum physics, as being more fundamental than classical physics, should not be subjected to the parallelism to classical theory implied in Lagrangian or functional quantization; in this he echoed Jordan's 1929 dictum [65] that QFT has to be formulated "without classical crutches"; in the present context this is done in terms of a concrete representation theoretical construction which is reminiscent of Wigner's method in the absence of interactions.

The reformulation of the analytic bootstrap-formfactor project for integrable

${ }^{28}$ Unlike quantum fields they are not (operator-valued) Schwartz distributions. 
models into a representation theoretical setting in terms of one-particle PFGs as generators of wedge-localized operator algebras constitutes a successful step in the adaptation of Wigner's idea to the realm of interactions. Although nothing is known about the solution of the inverse problem within the vast area of nontemperate (=nonintegrable) models (see later), one may ask whether among all the QFTs which can possibly be connected with a given unitary crossing symmetric S-matrix there is one for which the above idea of an analytic change of order permits a generalization.

To formulate such a problem one must first generalize the situation in [18] from one-particle PFGs to multi-particle states. This can be done by using the same concepts from modular localization, since the dense set of states of the modular Tomita operator $S$ also includes a dense sets of multi-particle states of arbitrary high particle number which form a basis in the Wigner-Fock Hilbert space of a QFTs with a complete particle interpretation. Whereas in a later section we will also consider interacting conformal QFTs which are known to have no particle interpretation and lead to a different kind of only partial integrability, in the present context "integrability" (without an additional specification) will always refer to the integrability in the sense of temperate particle wedgelocalized PFGs in QFTs describing particles. In any interacting QFT with a complete particle interpretation in terms of a incoming free field algebra $\mathcal{A}_{\text {in }}$ there holds the following theorem

Lemma 1 ([18]) Any state $|\psi\rangle \in \operatorname{dom} S_{\mathcal{A}(W)}=\operatorname{dom} S_{\mathcal{A}_{\text {in }}(W)}=\operatorname{dom} \Delta^{\frac{1}{2}}$ can be generated from the vacuum by operators $A$ and $A_{\mathcal{A}(W)}$ affiliated to each of the two algebras

$$
\begin{aligned}
& |\psi\rangle=A|0\rangle=A_{\mathcal{A}(W)}|0\rangle, A \in \mathcal{A}_{i n}(W), A_{\mathcal{A}(W)} \in \mathcal{A}(W) \\
& A \stackrel{\text { bijec }}{\leftrightarrow} A_{\mathcal{A}(W)} \text { closed, af filiated to } \mathcal{A}_{\mathcal{A}(W)}
\end{aligned}
$$

Here the second line defines the "emulated" operators $A_{\mathcal{A}(W)}$ as the image of wedge-localized free field operators under the bijection; the Reeh-Schlieder property of the commutant algebras together with modular operator theory (previous section) insures the denseness of their domains and their closability (for their closure the same notation will be maintained); the unique affiliation with $\mathcal{A}(W)$ is clinched by the appropriately formulated commutativity of the closed $A_{\mathcal{A}(W)}$ with the commutant $\mathcal{A}(W)^{\prime}$. It is precisely this properly defined commutativity with the commutant of the interacting algebras which insures the affiliation of the emulats to the interacting algebra of the chosen model and not to any other interacting algebras which happens to have the same particle content. Modular localization of states is much weaker than localization of operators, the two concepts only meet in the absence of interactions.

The crucial question is: how much does one have to know about the structure of the interacting algebra $\mathcal{A}(W)$ in order to construct the emulats; is the knowledge of the S-matrix in $J=S_{\text {scat }} J_{0}$ already enough for their explicit construction? We know that for integrable models the particle conserving twoparticle scattering functions determine the Z-F algebra and hence the QFT 
model, but can one also expect such a situation for nonintegrable models when $S_{\text {scat }}$ describes particle creation? We will present some consistency arguments which support such an idea, but are still far away from a proof. These arguments involve quite novel ideas and so we hope that the reader will find it sufficiently interesting to follow them.

Note that these unbounded emulats, whose unique existence in a given interacting theory with a complete particle interpretation is guaranteed, do not by themselves form an algebra since they neither can be multiplied (even in cases where this is possible $\left.(A B)_{\mathcal{A}(W)} \neq A_{\mathcal{A}(W)} B_{\mathcal{A}(W)}\right)$, nor does the formation of emulats commute with taking adjoints $\left(A^{*}\right)_{\mathcal{A}(W)} \neq\left(A_{\mathcal{A}(W)}\right)^{* 29}$. Although the original algebra cannot be reconstructed directly, the objects obtained from polar and spectral decompositions of the $\mathcal{A}(W)$ affiliated emulats can be used for its construction.

The hope that $\mathcal{A}(W)$ can be reconstructed solely from $S_{\text {scat }}$ is based on the before mentioned idea of an analytic ordering change. An important preparatory step is the formulation of the KMS propery in terms of emulats and its relation to the crossing property of formfactors. For this it is convenient to introduce the following notation, for simplicity we stay in $d=1+1$ and assume that the fields and the test-functions are real. With

$$
\begin{aligned}
& A\left(f_{1}, . f_{n}\right) \equiv: A\left(f_{1}\right) \ldots A\left(f_{n}\right):, A\left(f_{1}, . f_{n}\right) \text { affiliated to } \mathcal{A}_{0}(W), \text { supp } f_{i} \subset W \\
& A\left(f_{1}, . f_{n}\right)|0\rangle=\left|\check{f}_{1}, .\right\rangle_{i n}, \quad A(f)=\int a^{*}(\theta) \check{f}(\theta) d \theta+h . c ., p=m(\operatorname{ch} \theta, \operatorname{sh} \theta)
\end{aligned}
$$

denoting Wick-products of wedge-smeared free fields, these operators applied to the vacuum create n-particle states in momentum space wave functions $\check{f}$ which are the mass shell restrictions of the Fourier transform of the $f$ in terms of rapidity variables $\theta$. With $B$ denoting a generic operator in the free field algebra $B \in \mathcal{A}_{0}(W)^{30}$ and $A\left(f_{1}, . . f_{k}\right)$ affiliated Wick-ordered composites, the KMS relation (section 4) in a (for later purposes) useful form for the product of three operators reads

$$
\left\langle B A\left(f_{n}, . f_{l+1}\right) A\left(f_{l}, . f_{1}\right)\right\rangle \stackrel{K M S}{=}\left\langle A\left(f_{l}, . f_{1}\right) \Delta B A\left(f_{n}, . f_{l+1}\right)\right\rangle, \text { B composite } \in \mathcal{A}_{0}(W)
$$

where the existence of the analytically continued boost $\Delta=e^{-2 \pi K_{W}},\left(K_{W}=\right.$ generator of $\mathrm{W}$-preserving Lorentz boost) inside the right hand correlation functions is guaranteed by modular theory or by explicit calculation within the free field Wick ordering formalism. Applying the $A\left(f_{l}, . . f_{1}\right)$ on the right hand side of (21) to the bra vacuum, the wave functions inside the bra vector are the complex conjugate of the $\check{f}$, which in turn (using the analyticity resulting from the wedge localization) are identical to the analytically continued original wave functions.

\footnotetext{
${ }^{29}$ In fact it is easy to see that $([18])\left(A_{\mathcal{A}(W)}\right)^{*}|0\rangle=S_{\text {scat }} A|0\rangle$

${ }^{30}$ For free fields the operator algebra is generated by exponential Weyl operators.
} 
Finally by absorbing $\Delta^{\frac{1}{2}}$ of the $\Delta$ into the analytic continuation of the complex conjugate antiparticle wave functions (here the localization is important) we arrive again at the original wave functions:

$$
\iint \frac{d p_{1}}{2 p_{01}} . . \frac{d p_{1}}{2 p_{0 n}} \check{f}_{1}\left(p_{1}\right) . . \check{f}_{n}\left(p_{n}\right)\left\{\left\langle 0|B| p_{1}, . p_{n}\right\rangle-\left\langle p_{1}, . p_{l}\left|\Delta^{\frac{1}{2}} B\right| p_{n}, . p_{l+1}\right\rangle_{\text {c.o. }}\right\}=0
$$

$$
\left\langle 0|B| p_{1}, . p_{n}\right\rangle=\left\langle p_{1}, . p_{l}\left|\Delta^{\frac{1}{2}} B\right| p_{n}, . p_{l+1}\right\rangle=:\left\langle-p_{1}, .-p_{l}|B| p_{n}, . p_{l+1}\right\rangle, p_{i} \neq p_{k}
$$

where the subscript c.o. (contraction omitted) correspond to the omission of delta-function terms from Wick-contractions on the left hand side of (21), have been omitted. The free field distributions inside the curled bracket are actually square integrable; so the first line can be extended to all square integrable wave functions which then results in the second line. It is easy to see that in the presence of antiparticles the only change in the above relation is the assignment of the bra-momenta to antiparticles, in short $p \rightarrow \bar{p}$.

The interacting analog of the free KMS relation for modular wedge localization is

$$
\begin{aligned}
& \left\langle B A^{(1)}{ }_{\mathcal{A}(W)} A^{(2)} \mathcal{A}(W)\right\rangle \stackrel{K M S}{=}\left\langle A^{(2)}{ }_{\mathcal{A}(W)} \Delta B A^{(1)} \mathcal{A}(W)\right\rangle \\
& \left\langle B A^{(1)}{ }_{\mathcal{A}(W)} A^{(2)}\right\rangle=\left\langle A^{(2)} \mathcal{A}(W) \Delta B A^{(1)}\right\rangle= \\
& =\left(S_{\text {scat }} A^{(2) *} \Omega, \Delta B A^{(1)} \Omega\right), \text { since }\left(A^{(2)} \mathcal{A}(W)\right)^{*} \Omega=S_{\text {scat }} A^{(2) *} \Omega
\end{aligned}
$$

Rewriting this relation in terms of emulats for multiparticle states and using the fact that the emulats acting on the vacuum are multiparticle states, one obtains a pre-form of a particle crossing relation (again all contractions omitted) in which the particle content of the emulat in the middle of the left hand side is still unknown

$$
\begin{aligned}
& \langle 0| B\left(A^{(1)}\left(\check{f}_{1}, \check{f}_{l}\right)_{\mathcal{A}(W)}\left|\check{f}_{l+1}, \check{f}_{n}\right\rangle_{\text {in }}\right. \\
& =\int \check{f}_{1}\left(p_{1}\right), . . \check{f}_{l}\left(p_{l}\right) \frac{d p_{1}}{2 p_{01}} . . \frac{d p_{n}}{2 p_{0 n}} \text { out }\left\langle-\bar{p}_{n}, .-\bar{p}_{l+1}\left|\Delta^{\frac{1}{2}} B\right| \check{f}_{1} . . \check{f}_{l}\right\rangle_{\text {in }} \\
& \curvearrowright \int . . \int \frac{d p_{1}}{2 p_{10}} . \frac{d p_{n}}{2 p_{n 0}} \check{f}_{1}, . . \check{f}_{n}\left\{\langle 0 | B \left(A^{(1)}\left(p_{1}, . p_{l}\right)_{\mathcal{A}(W)\left|p_{l+1}, . p_{n}\right\rangle_{\text {in }}-}\right.\right. \\
& \left.\quad-{ }_{\text {out }}\left\langle-\bar{p}_{n}, .-\bar{p}_{l+1}\left|\Delta^{\frac{1}{2}} B\right| p_{1} . . p_{l}\right\rangle_{\text {in }}\right\} \quad=0
\end{aligned}
$$

In the sequel it will be shown how an extension of the idea of "analytic ordering change" solves this problem and also suggests a formula for formfactors of emulats as bilinear forms between multi-particle states. As in the integrable case, we assume that the vacuum formfactor is locally analytic. The presence of cuts resulting from inelastic multiparticle thresholds prevents such formfactors 
to be boundary values of meromorphic functions as in the integrable case, but these threshold cuts do not force them to be worse than locally square integrable. In this case we may pass from wedge-localized analytic wave function to nparticle wave functions with ordered supports and obtain (using the density of the $\check{f}$ in the space of the locally $L^{2}$-integrable wafe functions)

$$
\begin{aligned}
& \left\langle 0\left|B\left(A^{(1)}\left(\theta_{1}, . . \theta_{l}\right)\right)_{\mathcal{A}(W)}\right| \theta_{l+1}, . \theta_{n}\right\rangle_{i n} \equiv\left\langle 0|B| \theta_{1}, \ldots \theta_{n}\right\rangle_{i n} \\
& \text { for } \theta_{1}>\theta_{2} \ldots>\theta_{n}
\end{aligned}
$$

without having to know anything about the result of analytic order changes within the $\theta_{1}, . . \theta_{l}$ cluster and relative to the remaining $n-l$ particle cluster. In contradistinction to the integrable case, analytic changes through the multiparticle threshold cuts will inevitably be path-dependent, in particular there will be no analytic representation of the permutation group ${ }^{31}$.

Our free field illustration (22) suggests that the desired algebraic structure should retract to the Wick-formalism in case $S_{\text {scat }}=1$ and to the integrable analytic formalism for purely elastic 2-particle scattering. So we are looking for a formula which describes the action of a PFG (a one-particle emulat for simplicity) on an n-particle state in terms of a sum of terms in which the $\theta$-dependent creation- and annihilation- components pass through a particle cluster in order to arrive at its natural ordered position. Assuming ... $\theta_{k-1}>\theta_{k}>\theta>\theta_{k+1}>\ldots$ we seek a formula for the action of the distributional rapidity space creation component $C(\theta)$ of the PFG $A(\check{f})_{\mathcal{A}(W)}$ of the type

$$
\begin{aligned}
& C(\theta)\left|\theta_{1}, . ., \theta_{k}, \theta_{k+1}, . . \theta_{n}\right\rangle= \\
& \sum_{l} \int d \vartheta_{1} . . \int d \vartheta_{l} F_{\theta_{1} . . \theta_{k}, \theta}\left(\vartheta_{1}, . . \vartheta_{l}\right)\left|\vartheta_{1}, . . \vartheta_{l}, \theta, \theta_{k+1}, . . \theta_{n}\right\rangle
\end{aligned}
$$

where the $F$ only depends on the $\theta_{i}$ which are bigger than $\theta$ (i.e. which have been passed to achieve the natural order). If $\theta$ is already larger than all the others $\theta_{i}$ in the state, the creation part simply adds a particle. If $C(\theta)$ has to pass through a k-cluster to arrive at its ordered position the result is required to be of the above form whereas the annihilation component $C(\theta+i \pi)$ of $A(\check{f})_{\mathcal{A}(W)}$ leads to a delta function $\delta\left(\theta-\theta_{k}\right)$ multiplied with an integrand $F_{\theta_{1} . . \theta_{k-1}, \theta+i \pi}\left(\vartheta_{1}, . . \vartheta_{l}\right)\left|\vartheta_{1}, . . \vartheta_{l}, \theta_{k+1}, . . \theta_{n}\right\rangle$. The sum over 1 takes into consideration that processes of passing through particle clusters create and annihilate particle states of arbitrary high particle number, whereas the $\theta_{k+1} . . \theta_{n}$ on the right of the k-cluster remain unchanged.

The second requirement is that $F$ should not contain more detailed information about the interacting algebra $\mathcal{A}(W)$ than those contained in $S_{\text {scat }}$ which enters the wedge localization as a relative modular invariant. If the emulats depend on more detailed properties of $\mathcal{A}(W)$, they remains outside the range of the present method.

\footnotetext{
${ }^{31} \mathrm{~A}$ similar case in $\mathrm{x}$-space occurs if one extends a $\mathrm{d}=1+2 \mathrm{Wightman}$ setting to fields with braid.group statistics.
} 
The still undetermined $F$ will now be specified in terms of the "grazing shot" amplitude

$$
\begin{aligned}
F_{\theta_{1} . . \theta_{k}, \theta}\left(\vartheta_{1}, . . \vartheta_{l}\right) & =\sum_{s} \int d \chi_{1} . . \int d \chi_{s} S^{*}\left(\chi_{1}, . . \chi_{s} \rightarrow \vartheta_{1}, . . \vartheta_{l}\right) \\
\cdot S\left(\theta_{1}, . . \theta_{k}, \theta\right. & \left.\rightarrow \chi_{1}, . . \chi_{s}, \theta\right)
\end{aligned}
$$

which consists of a product of a scattering amplitude, in which one particle with rapidity $\theta$ scatters together with $k$ other particles such that it remains unchanged (second line). This is multiplied with the complex conjugate of a second amplitude whose purpose is to compensate all processes which would have occured even in the absence of grazing shot rapidity $\theta$. Hence without the presence of $\theta$ nothing happens i.e. $F$ reduced to (particle matrixelements of) the unit operator. This construction allows an extension from one-particle PFGs to multi-particle emulats.

In case of integrable models the S-matrix does not change the particle number and the grazing shot S-matrix $F(26)$ reduces to a multiplication with a product of elastic S-matrices [11],

$$
S\left(\theta-\theta_{1}\right) . . S\left(\theta-\theta_{k}\right)
$$

where in the case of the action of the annihilation components one S-factor is replaced by a delta contraction. In fact the whole idea behind the construction is to obtain a formula for a general S-matrix which in the integrable case passes to the known expression.

In the nonintegrable case this presentation is extremely formal since we know from [18] that nontemperate PFGs are only meaningful as wedge-localized $A(f)_{\mathcal{A}(W)}$ acting on wedge-localized states. Hence it is better to think of the PFGs as bilinear forms between particle states

$$
\left\langle\theta_{1}^{\prime}, . . \theta_{m}^{\prime}|C(\theta)| \theta_{1}, . . \theta_{n}\right\rangle
$$

and restore the localizing wave functions which one needs for passing to operators $A(f)_{\mathcal{A}(W)}$ after having computed these formfactors of the $C(\theta)^{\prime} s$.

The rapidities are uniformization variables which remove the elastic threshold, so that formfactors of integrable models are meromorphic functions in the multi- $\theta$ plane. The general analytic ordering picture assumes that the only singularities are cuts from higher inelastic thresholds which are locally square integrable. But if locally square integrable rapidity wave functions are admitted, we may extend the validity of the KMS relation from wedge localized wave functions to wave functions with ordered square integrable supports. In this way the analytic ordering picture connects the formfactor crossing identity (including its analytic property) to the KMS crossing identity for formfactors (25)

$$
\begin{aligned}
& \left\langle 0|B| p_{1} \ldots p_{n}\right\rangle_{\text {in }}={ }_{\text {out }}\left\langle-\bar{p}_{l+1}, .-\bar{p}_{n}|B| p_{1}, . p_{l}\right\rangle_{\text {in }} \\
& \quad \text { for }\left(\theta_{1}, . . \theta_{1}\right)>\left(\theta_{l+1}, . . \theta_{n}\right)
\end{aligned}
$$


It is remarkable that the interacting crossing identity just looks like its free field counterpart, apart from the fact that the bra-vectors refer to outgoing particle. Its derivation is not affected by the fragile conceptual status of our grazing shot construction for the action of emulats on multi-particle states.

Its origin from the KMS property of wedge-localization shows that this property in the center of particle theory shares its conceptual roots with those of the thermal manifestation of localization (the Einstein-Jordan conundrum of subvolume fluctuations, the Unruh Gedankenexperiment and Hawking radiation from black hole event horizons). In particular it has no relation to the crossing in the dual model and string theory which results from crossing properties of (Mellin transforms of) conformal correlation functions (section 4). String theory is the result of a fundamental misunderstanding of the subtleties of causal localization.

Although the grazing shot Ansatz reduces to the Wick contraction formula in the absence of interactions and to relations obtained from the Z-F algebra in the integrable case, these are only rather weak consistency requirements. The crucial step in establishing its correctness is the verification of wedge-locality

$$
\left[J A(f)_{\mathcal{A}(W)} J, A(g)_{\mathcal{A}(W)}\right]=0, \operatorname{supp} f, g \subset W
$$

which, apart from the trivial case of vacuum and one-particle matrix elements, the author was unable to do. This property may also turn out be useful for a perturbative determination of of PFGs $A(W)_{\mathcal{A}(W)}$ which one expects to be analog the iterative use of causality in the Epstein-Glaser iteration for pointlike fields. If the divergence of the on-shell perturbation series would be related to the pointlike singular character of fields, one expects that on-shell perturbation theory for wedge-local operators should converge.

Hence the grazing shot construction still hangs in the air and it is presently not worthwhile to present this idea in more details. In fact the reason why this is mentioned here at all is that the author firmly believes that even incomplete or failed attempts on important problems in particle physics should not go unmentioned; reporting on them is not less important than presenting established facts. The proof of mathematical existence of nontrivial models outside the narrow setting of integrability and the discovery of controlled approximations remains still the paramount problem of QFT even after almost 90 years after its inception. The resounding observational success resulting from low orders of the (unfortunately) diverging perturbative series has in no way disburdened QFT from its conceptual-mathematical fragility; the idea that the low orders are an asymptotic solution in the limit of vanishing interaction strength remains an unproven conjecture, even after Dyson pointed to this problem more than half a century ago.

In the integrable case the inverse problem has a unique solution, but there is no argument that excludes the possibility that those elastic S-matrices also admit non-integrable solutions. The lack of a uniqueness proof even includes the absence of interactions in the sense of $S_{s c a t}=1$, i.e. the question whether besides the massive free field, the Hilbert space can accommodate other local 
nets with the same representation of the Poincare group and the TCP operator (and hence the same modular data ${ }^{32}$.

Without additional restrictions it is in fact quite easy to construct a continuous infinity of covariant wedge-localized algebras. Any unitary operator in $H$ which preserves the vacuum and commutes with the wedge-preserving Lorentz boost and the reflection $J_{0}$ on the edge of the wedge of the form

$$
V=e^{i \eta}, \eta=\sum \frac{1}{n !} \int \tilde{\eta}\left(x_{1}, . . x_{n}\right): A\left(x_{1}\right) . . A\left(x_{n}\right): d x_{1} . . d x_{n}
$$

will lead to a net of wedge algebras with $S_{\text {scat }}=1$ which, apart from the special case that $V$ implements an automorphism of $\mathcal{A}_{0}(W)$, is inequivalent to the net generated by a free field. The restrictions on the coefficient functions in the rapidity parametrization $\tilde{\eta}\left(\theta_{1}, . . \theta_{n}\right)$ for $\mathrm{d}=1+1$ resulting from the shared modular data are very mild: the coefficient functions $\tilde{\eta}$ can only depend on $\theta$ differences, the $J_{0}$-invariance leads to a reality condition and $V|0\rangle=|0\rangle$ requires the absence of terms with only creation operators.

The passing from a fixed $W$ to the net of wedges $W_{x}(x=$ apex $)$ is achieved by applying translations $U(x)$

$$
\mathcal{A}\left(W_{x}\right) \equiv V_{x} \mathcal{A}_{0}\left(W_{x}\right) V_{x}^{*}, V_{x}=U(x) V U(x)^{*}
$$

Unlike the previous method which was based on the temperate/nontemperate dichotomy of emulats, it would be very difficult to separate integrable models with $S_{\text {scat }}=1$ from this huge set of possibilities ${ }^{33}$.

This argument only concerns the connection between $S_{\text {scat }}=1$ and the family of wedge-localized local nets. In order to arrive at the full net, one still has to intersect wedge algebras in order to obtain compact localized double cone algebras $\mathcal{A}(\mathcal{D})$ and one knows from the work of Lechner [4] that the requirements about the cardinality of phase space degrees of freedom, which insures the nontriviality of intersections, are extremely restrictive; in fact most wedge nets will not possess nontrivial double cone nets. This still leaves the possibility that the looked for uniqueness may arise in the problem of forming intersections.

The aim of the present approach based on wedge-localization is closely related to the old S-matrix setting; it shares with the ideas behind the abandoned S-matrix bootstrap and Mandelstam's later attempts (to use spectral representations for the description of analytic properties of elastic scattering amplitudes $^{34}$ ) its proximity to laboratory observables. The S-matrix (formfactor of the identity) and formfactors are on-shell objects (correlations restricted to the mass-shell) which are directly accessible to experiments. They contain much more information than Lagrangians which lead to a unique perturbative series.

\footnotetext{
${ }^{32}$ In [66] it was shown that as a consequence of Huygens principle, conformal QFT leads to the uniqueness of the inverse problem for $S_{\text {scat }}=1$.

${ }^{33}$ The consistency of this method with the emulation construction may lead to further restrictions.

${ }^{34}$ It is however incompatible with dual model and string theory constructions and their S-matrix interpretations.
} 
The constructions in this section are thought of as a first step to construct the full (off-shell) QFT.

This makes them top-to-bottom approaches in the sense that one starts with a list of well-understood properties, which one expects a QFT to describe, and then sets up the mathematics to understand their consequences. Pointlike localized fields and their correlations are far removed from particles and their on-shell manifestations; they form the bottom-to-top setting of Lagrangian or functional quantization; in a mass-shell based top-to-bottom approach they are only expected to appear at the end (in case they are still needed). In quantization approaches one follows a translation dictionary which forces a more fundamental QFT to follow formal rules which correspond to those of a less fundamental classical theory. As a result, the physical interpretation and content only emerges at the end of perturbative calculations and there is hardly any mathematical controll about what one is doing. A top to bottom approach as the present attempt cuts all classical connections and replaces them by a tighter conceptual-mathematical control.

Perhaps the conceptually most useful analogy to what is done here is to consider it as an extension of the Wigner representation theoretical approach to the presence of interactions. The functorial connection between Wigner particle and free fields breaks down in the presence of any interaction ${ }^{35}$. The relation between particles and their free fields to interacting wedge-localized emulats can be viewed as its substitute. This much weaker particle-field connection makes QFT more fundamental and at the same time computationally more inaccessible than QM.

\section{Conformal integrability}

There exists a different notion of "kinematic" integrability which is not directly related to the dynamics of a model, but rather refers to a discrete combinatorial structure of its countable superselected localizable charge sectors which the DHR superselection theory [21] uniquely associates to a local (neutral and invariant under inner symmetries) observable algebra. More explicitly, it refers to the structure of the set of equivalence classes of localizable representations of the observable net $\{\mathcal{A}(\mathcal{O})\}_{\mathcal{O} \in R^{4}}$ given in its vacuum representation. In the case of massive theories with Bose/Fermi statistics this structure turns out to have the form of a tracial state on a discrete (type $\mathrm{II}_{1}$ ) operator algebra which contains the infinite permutation group algebra whose representation theory is responsible for the particle/field statistics. In fact this algebra turns out to be the dual of a compact "internal symmetry" algebra which commutes with the Poincaré group [21] [67]. The internal symmetry group acts on a larger uniquely determined "field algebra" which contains the observable algebra as a fixed point algebra under the action of the symmetry group [67]. This construction demystifies the concept of inner symmetries, which dates back to Heisenberg's

\footnotetext{
${ }^{35}$ This is different in QM where the interacting Schrödinger equation remains functorially related to its operator Fock space formulation.
} 
phenomenological introduction of isospin into nuclear physics. Its conceptual origin in QT is the principle of causal localization of quantum observables and their superselected localized representations which can be combined into a field algebra on which a compact group acts ${ }^{36}$. Although it has no counterpart in classical physics, for the application of the method of Lagrangian or functional quantization it is necessary to read this property back into the classical setting.

In previous sections we have seen that causal localizability results in vacuum polarization, thermal manifestations and associated intrinsic ensemble probabilities. The DHR theory adds the superselection theory and inner group symmetries of field algebras in which the observable algebras are embedded as fix-point algebras under the action of the internal symmetry to this list.

The relation between local observables and their extension into a field algebra becomes particularly interesting in theories which cannot be described in terms of Lagrangian quantization; the most prominent family of such theories are conformally invariant $\mathrm{QFTs}^{37}$. In that case the observable algebras obey the Huygens principle i.e. the vanishing of (graded) commutators of pointlike fields also for timelike distances. Apart from chiral conformal theories which live on lightlike lines so that the distinction between space- and time- like disappears and for which the observable algebras (current or energy-momentum algebras) as well as the generators of their representations (braid group commutation relations) in many typical cases can be explicitly constructed, the "Huygens" observable algebras in higher dimensions are more complicated and do not seem to be integrable.

However the anomalous spectrum of scale dimension in conformal theories seems to be susceptible to systematic classification. This is because it is defined in terms of the phases of a unitary operator, the generator of the center $Z$ of the conformal covering group. Whereas the "Huygens observable algebra" lives on the compactified Minkowski spacetime $M^{c}$, the field algebra, which is generated by pointlike fields with anomalous dimensions, is localized on its universal covering $\widetilde{M^{c}} 38$. As in the simpler case of chiral models, where the nontrivial center is closely related to the issue of plektonic statistics (braid-group commutation relations of fields), one is accustomed to view such properties as being more on the kinematic than on the dynamic side, although such distinctions become somewhat blurred outside that kind of QFT which is used for the description of particles to which conformal QFT definitely does not belong. In the sequel we will argue that the the spectrum of anomalous dimensions is indeed accessible to rigorous classification and that therefore the terminology kinematical (or better "partial") integrability of conformal QFTs is quite appropriate. Integrability without additional specification will be reserved for the full dynamical integrability which is limited to $\mathrm{d}=1+1$ as explained in the previous section.

\footnotetext{
36 To put it bluntly: compact groups arise from quantum causal localization in the presence of mass gaps [21].

${ }^{37}$ The indirect way of interpreting conformal theories as massless limits of perturbatively accessible massive models was only successfull in the case of the massive Thirring model for which the perturbative Callen.Symanzik equation comes with a vanishing beta function [46]..

${ }^{38}$ Equivalently they can be interpreted as operator-valued distributional sections on $M^{c}$.
} 
The method to investigate partial integrability in conformal QFT is nonperturbative and, as the constructive approach to integrable massive models, uses representation theory in particular the representation theoretical methods of the DHR superselection theory. Already for the low-dimensional chiral models the role of the infinite permutation group $\mathbf{P}_{\infty}$ is taken over by the much richer representation theory of the braid group $\mathbf{B}_{\infty}$. As the DHR theory led to tracial states ("Markov traces") on the infinite permutation group, chiral conformal theories require a classification of representations associated with tracial states on $\mathbf{B}_{\infty}$.

Tracial states on combinatorial algebras in a much more general context are an important tool in Vaughn Jones subfactor theory [24]. The partial integrability of the braid group representation structure, even in cases where it was not possible to compute n-point correlation functions of observable fields ${ }^{39}$ led to a mutually interesting and fruitful connection with subfactor theory (which may be viewed as a vast extension of group representation theory).

Whereas $d \geq 1+2$ interacting models with a complete particle interpretation are always non-integrable (previous section), their superselection structure which forms a discrete tracial algebra, is by definition integrable since tracial states on words in extended group algebra of the permutation $\mathbf{P}_{\infty}$ or braid group $\mathbf{B}_{\infty}$ are computable by combinatorial methods. This is of particular interest in higher dimensional conformal theories for which DHR superselection setting suggests the relevance of a braid-permutation group $\mathbf{P} \mathbf{B}_{\infty}$ whose representation-theoretical studies are still in their its infancy [23].

To investigate this partial integrability one needs to know some structural properties of conformal QFTs, in particular that anomalous dimensions are labels of superselection sectors of observable algebras. The braid group and the spacetime covering aspect arises through the time-like Huygens structure, whereas the permutation group enters as usual through spacelike commutativity; but the $\mathbf{P B}_{\infty}$ group is not simply a product of its two subgroups! A detailed knowledge about local observables is not required; it is not necessary to know what is "inside" each superselection sector, the rules of their compositions and decompositions suffice. The combinatorial algebras of the Hecke or BirmanWenzl type are typical algebraic structures which arise in this context [75].

There have been some misconceptions in the recent literature about the status of conformal QFT within particle theor ${ }^{40}$. Conformal QFT was first proposed in the beginning of the $60 \mathrm{~s}$, but as a result of their remoteness from particle theory in particular scattering theory the interest in them waned quickly. Most of the intuitive arguments against their direct use in particle theory were later made rigorous. Here are some of them

1. A conformal field with canonical (free field) short distance behavior is inevitably identical to a free field theory [69].

\footnotetext{
${ }^{39}$ A notable exception is the chiral Ising QFT [68].

${ }^{40}$ In many contemporary articles the fact that the tree-approximation of conformal theory (isomorphic to the classical structure) allow a restriction to a zero mass shell has been used to incorrectly allege that they can describe quantrum particles in the sense of scattering theory and the S-matrix.
} 
2. A conformal QFT cannot be perturbatively constructed from free massless fields and the perturbative behavior of massive renormalizable $\mathrm{d}=1+3$ models (contrary to some models in $d=1+1^{41}$ ) is not "soft" in a sense which would allow to take a massless limit within the perturbative Lagrangian setting.

3. The LSZ scattering limits of interacting conformal fields vanish ${ }^{42}$.

The proof for 1 . and 3 . is actually quite simple, the first follows from the fact that the canonicity of scale dimension requires a free field behavior for short distances which in conformal theories implies the freeness of the field itself. The third is a consequence of the fact that the increase of the short distance dimension above its smallest possible value allowed by positivity (that for a free field) automatically reduces the singularity at the zero mass shell $p^{2}=0$ which then is too weak to match the dissipating behavior of wave packets which would be necessary in order to arrive at a nontrivial LSZ limit. The QED prescription, which interprets photon-inclusive cross sections as the observable manifestations of charged particle does not work for assigning a particle interpretation to conformal theories.

The airy use of conformal QFTs in many recent publication shows that particle physics is in the process of loosing its history since none of the old arguments showing the problematic relation of conformal QFT with particle theory has been addressed. It is not forbidden to think about conformal theories of resulting from massive theories in a hypothetical limit in which all particle creation threshold fall on top of each other, but as long as there is no such massive theory with a perturbative Callan-Symanzik equation with $\beta=0$ (see remarks in section 3) this is of not much use.

Although conformal theories play no direct role in particle theory, their apparent mathematical simplicity make them ideal "theoretical laboratories" for the study of structural problems of QFT. Conformal transformations relate compact to non-compact regions and in this way extends the concept of modular localization. Low dimensional chiral theories were the first theories for which representation theoretical nonperturbative methods led to proof of existence as part of their explicit construction before such methods were also successfully applied for massive integrable models (previous section). They played an important role in the adaptation of the Tomita-Takesaki modular theory of operator algebras to problems of modular localization, and led to a fruitful meeting of minds between algebraic QFT and the subfactor theory.

An important step in the history of conformal QFT was the understanding of the role of the Huygens principle in the definition of conformal observables and anomalous dimension-carrying charged fields which led in 1975 to a conformal decomposition theory [70][71][22]. There were two viewpoints about conformal

\footnotetext{
${ }^{41}$ The most prominent exeption is the massive Thirring model. In fact the suspicion that $\beta(g) \equiv 0$ which led the derivation of the Callan-Symanzik equation to all orders [46] came from the observation of softness in $\mathrm{m} \rightarrow 0$.

${ }^{42}$ The Hilbert space positivity forces the Källén-Lehmann spectral measure to have a singularity which is milder than a mass-shell delta function.
} 
invariance; one can either say that conformal fields "live" (are univalued) on the universal covering of the compactified Minkowski spacetime $\widetilde{M^{c}}$, or that they are distribution-valued sections on $M^{c}$. In the first case [22] (which probably goes back to Irving Segal) one encounters infinitely many "heavens" above and "hells" below $M^{c}$ and there exists a generator of the center of the universal conformal covering group $Z \in \widehat{S O(4,2)}$ (for $\mathrm{d}=1+3)$ such that $Z^{n}$, $n$ integer numbers those heavens and hells and $n=0$ corresponding to the compactification $M^{c}$ of our living spacetime. The center is a certain conformal rotation at the angle $2 \pi$ whose spectrum results in the formula spec $Z=\left\{e^{i 2 \pi d_{\alpha}}\right\}$ where $d_{\alpha}$ runs over the (anomalous) conformal field dimensions.

There is an analogy of this situation to the physics of plektons in $d=1+2$. In this case the Poincaré group $\mathcal{P}$ has an infinite covering $\widetilde{\mathcal{P}}$, but the spacetime has none. The Wigner-Bargmann representation theory of positive energy representations in $d=1+2$ however creates a kind of covering due to the semiinfinite string-like nature of the plektonic wave functions [39]. Apart from that difference the anomalous spatial spin corresponds to the anomalous dimension and the plektonic statistics (anyons are abelian plektons) resembles an imagined "timelike braided exchange", with the resulting statistical phase [21] corresponding to the eigenvalue of $Z$. For a long time it was suspected that there is some kind of free or at least integrable plektonic QFT associated with the Wigner-Bargman representation, but meanwhile this idea has been disproven [56].

The prerequisite for conformal observables is that their scale dimension is integer $^{43}$; typically their pointlike generators are conserved currents or the energymomentum tensor which result from the "localization" of global symmetries; but in principle any field with integer dimension satisfies the Huygens propertyn and hence can be included into the observables. Such local fields live on $M^{c}$ and commute with the center $Z$ of the conformal group $S O(4,2)$. As a result their commutators are concentrated on the mantle of the light cone which in turn implies that their correlations functions are multivariable rational analytic functions [72]. Despite their simple appearance, nontrivial $d=1+3$ Huygens fields have not yet been constructed and the problem of their integrability remains unresolved.

The anomalous dimensions play the role of generalized superselected charges carried by the anomalous dimensional fields ${ }^{44}$. The application of the spectral decomposition theory with respect to the center $Z$ leads to the following decomposition of fields

$$
\begin{aligned}
& A(x) \rightarrow A_{\alpha, \beta}(x) \equiv P_{\alpha} A(x) P_{\beta}, Z=\sum_{\alpha} e^{i 2 \pi d_{\alpha}} P_{\alpha} \\
& A_{\alpha, \beta}(x) B_{\beta, \gamma}(y)=\sum_{\beta^{\prime}} R_{\beta, \beta^{\prime}}^{(\alpha, \gamma)}(x, y) B_{\alpha, \beta^{\prime}}(y) A_{\beta^{\prime}, \gamma}(x)
\end{aligned}
$$

\footnotetext{
${ }^{43}$ For semiinteger dimension as they already occur for free spinors it is necessary to take the double covering of $M_{c}$. These fields fulfill an extended Huygens principle on the double covering.

${ }^{44}$ The analogy works better with squares of charges since the matter-antimatter charge compensation has no counterpart the composition of anomalous dimensions.
} 
The R-matrices depend discontinuously on spacetime, they are locally constant but different for time- and space- like separations. For time-like separation the distinction positive/negative timelike is topologically similar to left/right distinction in chiral theories.

These decompositions appear first for abelian (anyonic) R-matrices in [70]; only after the path-breaking work in the 80s by Belavin,Polyakov and Zamolodchikov [73] they were generalized to the nonabelian braid group (plektonic) representations which appear in the exchange algebras of chiral models [75][76]. Although (apart from free fields) chiral conformal field theories do not describe particles, it is customary to refer to the quanta, which carry a discrete representation of the conformal rotation and lead to R-matrix commutation relations of the Artin braid group, as "plektons".

The topological similarity of the positive and negative time-like Huygens region leads one to expect the anomalous dimension spectra to be connected with braid group representations. But since there is also the requirement from spacelike commutation which leads to the nontrivially combined $\mathbf{B} \mathbf{P}_{\infty}$ group (the "words" in $\mathbf{B}_{\infty}$ intertwine nontrivially with those in $\mathbf{P}_{\infty}$ ), the kind of $\mathbf{B}_{\infty}$ representations as they occur in chiral theories (Hecke-, Birman-Wenzl algebras,..) are not expected to re-appear in this form in higher dimensional CFT. Some of the new problems were mentioned in [69].

It is not difficult to write the defining relation between the $b_{i}, t_{i}$ generators of $\mathbf{B} \mathbf{P}_{\infty}$ [23]. The $Z$ spectrum of any 4-dim. conformal model should belong to one representation of these relations. But unlike in the chiral case, where the exponential Bose field model was available a long time before the later systematic construction of families of chiral models in the work of [73], there exists presently no illustrative nontrivial example; the conformal invariant generalized free field (which results from the AdS free field by applying the AdS-CFT correspondence) is too far away from physical fields ${ }^{45}$ in order to be of much interest. Recently proposed dimensional spectra on the basis of analogies with those of transfer matrices of Ising lattice models [74] are not supported by the Huygens structure of conformal observables and their expected dimensional spectra from the $\mathbf{B P}_{\infty}$ representation structure.

The group theoretical origin of the simplification in $\mathrm{d}=1+1$ is the factorization of its conformal group $S O(2,2)=S L(2, R) \times S L(2, R)$ which leaves the 3 -parametric Moebius group as the space-time symmetry of a chiral theory on $\mathbb{R}$ or its compactification $S^{1}$ (with the possibility to extend it to Diff $\left(\mathrm{S}^{1}\right)$ ). The group theoretical factorization is followed by a decomposition of the $\mathrm{d}=1+1$ conformal observables into its chiral components living on separate light rays. The chiral theories have proven to be the most susceptible to the classification and construction of their their superselected representation sectors and sector creating plektonic fields; in particular for "rational" models (i.e. models with a finite number of representations) many explicit constructions are available [79].

The first illustrative model for the decomposition theory was the exponen-

\footnotetext{
${ }^{45}$ Its abundance of degrees of freedom leads to the before-mentioned pathological timelike causality properties and the absence of reasonable thermodynamik behavior.
} 
tial Boson field [70]. In this case the analogy of anomalous dimension with superselecting charges takes a very concrete form. In a somewhat formal way of writing

$$
\begin{aligned}
j(x) & =\partial_{x} V(x), \quad\left\langle j(x), j\left(x^{\prime}\right)\right\rangle \sim \frac{1}{\left(x-x^{\prime}+i \varepsilon\right)^{2}} \\
\Psi^{(q)}(x) & =e^{i q V(x)},\left[Q, \Psi^{(q)}(x)\right]=q \Psi^{(q)}(x), Q=\int j\left(x^{\prime}\right) d x^{\prime} \\
\Psi^{(q)}(x) & =\sum_{q^{\prime}-q^{\prime \prime}=q} \Psi_{q^{\prime}, q^{\prime \prime}}^{(q)}(x), \Psi_{q}^{q^{\prime}}(x) \equiv P_{q^{\prime}} \Psi_{q}(x) P_{q^{\prime \prime}}
\end{aligned}
$$

In the last line the $P_{q^{\prime}}$ are the projectors onto the continuous subspaces $H_{q^{\prime}}$ where $q^{\prime}$ runs over all continuous superselected charge values in a nonseparable Hilbert space. This model belongs to the class of non-rational chiral models, but by enlarging the observable algebra to include $\Psi^{(q)}(x)$ fields with $q^{\prime} s$ leading to integer scale dimensions $q^{2}$

$$
U(\lambda) \Psi^{(q)}(x) U(\lambda)^{*}=\lambda^{q^{2}} \Psi^{(q)}(\lambda x)
$$

the number of superselected sectors becomes finite (quantized charges) and the resulting model is "rational" and integrable.

The situation becomes especially interesting for an n-component current algebra.

$$
j_{k}(x)=\partial_{x} V_{k}(x), \quad k=1 . . n, \Psi^{(\vec{q})}(x)=e^{i \vec{q} \vec{V}(x)}
$$

In that case the maximal local extensions of the current algebra are classified in terms of integer n-dimensional lattices and their superselected sectors are characterized in terms of their dual lattices. In this setting the selfdual lattices correspond precisely to situations with a trivial superselecting structure (the vacuum sector is the only sector). The corresponding selfdual lattices correspond to the largest exceptional finite groups, the most mysterious among them is known as the "moonshine" group. The fact that quantum localization lead to such subtle group theoretic properties gives an impression of the conceptual depth and its many unexpected relation to other mathematical and physical concepts.

But conceptual subtleties can also lead to pertinacious misunderstandings. The most prominent arose from the picture of an "embedding" of this n-component current theory into its n-component inner symmetry space called target space of string theory. The idea was to convert the n-component inner symmetry space of the charge-carrying sigma-model $\Psi^{(\vec{q})}(x)$ fields into a "target" space which carries a non-compact group representation, specifically a positive energy representation of the Poincaré group.

It turns out that the infinitely many oscillators of a (supersymmetrically extended) current theory permit precisely one solution in form of the 10-parametric highly reducible superstring representation. But in order to achieve this one has to organize these oscillator degrees of freedom in a different way from that required by the modular localization on a chiral lightlike line. In fact already the 
spectrum of the multicomponent superselected chiral charge does not match the mass spectrum of the superstring representation (different zero modes) so that the chiral "source" theory and the "target representation" of the Poincare group live in different Hilbert spaces. The localization of a positive energy representation is a fait accompli and it is point- and not stringlike ${ }^{46}$. The relation of the chiral model and the Poincaré group acting in its putative target space neither support an embedding of a lower dimensional QFT into a higher dimensional one (which according to the holistic aspects of different modular localization is never possible in QFT) nor a stringlike localization.

In fact in the sense of loalization in Minkowski spacetime all the non zero oscillator degrees of freedom form a quantum mechanical oscillator chain in the inner space "on top of a localization point". One may call this a string (at the risk of creating confusions), except that in QM localization is not a spacetime-related intrinsic property but depends on what the working physicist wants to make out of it. Given the 50 year domination of string theory and the foundational role of causal localization in QFT these still ongoing misunderstandings represents certainly the deepest schism which ever occurred within particle physics.

The source-target terminology incorrectly anticipated that the relation between the chiral conformal QFT and ST can be understood as an embedding of the chiral source into the n-component target. Whereas such imbedding (and their Kaluza-Klein inversions) are perfectly possible in classical field theories and even in QM, this is not possible in QFT. The reason is that quantum causal localization is "holistic" whereas localization in classical field theory or quantum mechanics is not. The holistic organization of oscillators for implementing the localization of the currents and their associated sigma model fields on the lightlike line is simply not the same as that which comes with a positive energy representation and the related localization on the target.

The only memory which the target space use of the oscillators has about the chiral current model is that the mass spectrum from the superstring representation is (up to a scale-setting numerical factor) equal to the anomalous dimension spectrum of the conformal composites which appear in the converging global operator expansion of the operator product of two sigma model fields. This is connected to Mack's observation [77][78] that the dual model "masses" are obtained from the Mellin transform of global operator product expansions in conformal QFT. The would-be particle spectra in string theory (e.g. the superstring spectrum in $\mathrm{d}=1+9$ ) have their origin in anomalous dimension spectra of conformal QFT and the conformal origin of dual model crossing has no relation with the crossing in particle physics (section 5) and it is not comprehensible why the rarity of finding representations of the Poincaré group in a target space construction of a chiral theory (the superstring and its M-theoretic modifications are the only representations) should attach a fundamental significance which led string theorists to claim that we are living in a dimensionally reduced 10

\footnotetext{
${ }^{46}$ There are no string-localized infinite spin representation components in the reducible superstring representation.
} 
dimensional spacetime. The reductionist trend in QFT supports the idea that a foundational theory should not admit alternative realizations of the same underlying principle, but it does not suggest that rarity of a construction should attach a foundational significance to it.

One can learn a lot from corrections of incorrect ideas about the meaning and consequences of causal localization in ST. More extensive presentations of these misunderstandings about localization can be found in [80].

There are good reasons to also maintain a skeptic attitude with respect to all ideas which originated from string theory, even if afterwards they were presented within the setting of QFT as in the case of the AdS-CFT correspondence. In relations between QFTs in different dimensions the touchy point is the degrees of freedom issue and its implications for causal localization; this has simply no counterpart in classical field theory nor in quasiclassical approaximations. A QFT may fulfill local commutativity (Einstein causality) but violate the causal completion property as a result of having more degrees of freedom in the causal completion $\mathcal{A}\left(\mathcal{O}^{\prime \prime}\right)$ than there were in the original region $\mathcal{A}(\mathcal{O}) \varsubsetneqq \mathcal{A}\left(\mathcal{O}^{\prime \prime}\right)$. This contradicts our ideas of causal propagation; "poltergeist" degree of freedoms which enter the region of causal determination from nowhere should not occur in a physically acceptable theory. Their absence is a property of relativistic propagation of classical Euler-Lagrange equation and enters formally QFT through Lagrangian quantization; but in a general setting of QFT this has to be separately added (the time-slice property in [81]).

A typical example for a generating field which violates this causality requirement as the consequence of too many phase space degrees of freedom is the before mentioned appropriately chosen generalized free field (a free field with continuous mass distribution). But precisely such fields appears when one computes the conformal field which according to the AdS-CFT correspondence results from a AdS free field [82]. The algebraic derivation of the AdS-CFT correspondence shows that this phenomenon is intrinsic to this kind of correspondence. It has its counterpart in the opposite direction $C F T \rightarrow A d S$, where one finds that there are not enough degrees of freedom in order to support a physical causal AdS theory, as a result only non-compact localized algebra as $\mathcal{A}(W)$ are nontrivial whereas double cone algebras remain empty $\mathcal{A}(\mathcal{C})=\{\mathbb{C} 1\}$. The preservation of phase space degrees of freedom is closely related to the shared spacetime group symmetry. This physical shortcoming in no way has any influence on the mathematical existence of both sides.

These are verifiable structural facts; they do not permit any exception just like TCP or spin\&statistics. The AdS-CFT Maldacena conjecture, to the extend that it places a physically viable theory on both sides of the correspondence, contradicts these facts Even worse, results which once were known at times when the particle theory community was much smaller seem to have been irrevocably lost. No wonder that incorrect ideas about embedding of QFTs and dimensional reduction of extra dimensions enjoy a widespread popularity. In most publications the awareness that such problems cannot be addressed by manipulating Lagrangians but need the structural knowledge about interacting fields and their correlations has been lost. Often these misunderstandings arise from 
quasiclassical approximations thus overlooking the fact that they contain no informations about modular localization and the phase space degrees of freedom cardinality which sustains the causal completion property. This is especially evident in the notion of "branes". Their quasiclassical constructions do not show what really happens namely that all the degrees of freedom which were contained in the original physical QFT are compressed into the brane which, as a result of overpopulation, becomes unphysical [78].

One problem in which the relation between localization and cardinality of phase space degrees of freedom has been used to prove the existence of certain $\mathrm{d}=1+1$ integrable models is Lechner's work [4], in particular his theorem about the nontriviality of double cone intersections of wedges algebra based on the modular nuclearity property of the degrees of freedom resulting from the Z-F algebra structure of wedge generators.

Acknowledgment: I am indebted to Jens Mund and Jakob Yngvason for innumerous discussions on various topics which entered this work. Special thanks go to Detlev Buchholz for a critical reading of section 5 which led to its reformulation.

\section{References}

[1] V. I. Arnold, V.V. Koslov and A. I. Neishtadt, Mathematical Aspects of Classical and Celestial Mechanics, Springer Verlag 2002

[2] H. Babujian, A. Förster and M. Karowski, Nucl.Phys.B736, (2006) 169, arXiv:hep-th/0510062

[3] J. Glimm and A. Jaffe, Quantum physics: a functional integral point of view, Springer Verag 1981

[4] G. Lechner, An Existence Proof for Interacting Quantum Field Theories with a Factorizing S-Matrix, Commun. Mat. Phys. 227, (2008) 821, arXiv.org/abs/math-ph/0601022

[5] R.F. Dashen, B. Hasslacher and A. Neveu, Phys. Rev. D. 10, (1974) 4130

[6] B. Schroer, T.T. Truong and P. Weiss, Phys. Lett. B 63, (1976) 422

[7] M. Karowski and P. Weisz, Nucl. Phys. B 139, (1978) 445

[8] H-J Borchers, On revolutionizing quantum field theory with Tomita's modular theory, J. Math. Phys. 41, (2000) 860

[9] M. Karowski, H.J. Thun, T.T. Truong and P. Weisz, Phys. Lett. B 67, (1977) 321

[10] B. Berg, M. Karowski, V. Kurak and P. Weisz, Nucl. Phys. B 134 (1978) 125 
[11] H. Babujian, A. Fring, M. Karowski, A. Zapletal, Nucl.Phys. B538 (1999) 535

[12] A. B. Zamolodchikov and Al. B. Zamolodchikov, Ann. Phys. 120, (1979) 253

[13] H. Babujian and M. Karowski, Int. J. Mod. Phys. A1952, (2004) 34, and references therein to the beginnings of the bootstrap-formfactor program

[14] B. Schroer, Nucl. Phys. B 499, (1997) 547

[15] B. Schroer, Modular localization and the $d=1+1$ formfactor program, Annals of Physics 295, (1999) 190

[16] G. Lechner, J.Phys. A38, (2005) 3045

[17] J. Mund, An algebraic Jost-Schroer theorem, Commun. Math. Phys. 315, (2012) 445

[18] H. J. Borchers, D. Buchholz and B. Schroer, Commun.Math.Phys. 219 (2001) 125

[19] B. Schroer, The Einstein-Jordan conundrum and its relation to ongoing foundational research in local quantum physics, to be published in EPJH, arXiv:1101.0569

[20] A. Duncan and M. Janssen, Pascual Jordan's resolution of the conundrum of the wave-particle duality of light, arXiv:0709.3812

[21] R. Haag, Local Quantum Physics, Springer 1996

[22] M. Lüscher and G. Mack, Comm. Math. Phys. 41, (1975) 203

[23] R. Fenn, R. Rimányi and C. Rourke, The braid-permutation group, Topology 36, (1997) 123

[24] Vaughan F. R. Jones and V. S. Sunder, Introduction to subfactors, Cambridge University Press (1997) Volume 234

[25] R. Haag, Discussion of the 'axioms' and the asymptotic properties of a local field theory with composite particles (historical document), Eur. Phys. J. H $35,243-253$ (2010)

[26] R. F. Streater and A. S. Wightman, PCT SpinESStatistics and all that, New York, Benjamin 1964

[27] W. G. Unruh, Notes on black hole evaporation, Phys. Rev. D14, (1976) 870-892

[28] G. 't Hooft, Int. J. Mod. Phys. A11, (1996) 4623 
[29] B. Schroer, Causality and dispersion relations and the role of the S-matrix in the ongoing research, arXiv:1107.1374

[30] B. Schroer, A critical look at 50 years particle theory from the perspective of the crossing property, Found. Phys. 40, (2010) 1800, arXiv:0906.2874

[31] J. J. Bisognano and E. H. Wichmann, On the duality condition for quantum fields, Journal of Mathematical Physics 17, (1976) 303-321

[32] J. Mund, Commun. Math. Phys. 286, (2009) 1159, arXiv:0801.3621

[33] S. J. Summers, Tomita-Takesaki Modular Theory, arXiv:math$\mathrm{ph} / 0511034 \mathrm{v} 1$

[34] S. Weinberg, The Quantum Theory of Fields I, Cambridge University Press

[35] R. Brunetti, D. Guido and R. Longo, Modular localization and Wigner particles, Rev. Math. Phys. 14, (2002) 759

[36] L. Fassarella and B. Schroer, Wigner particle theory and local quantum physics, J. Phys. A 35, (2002) 9123-9164

[37] J. Mund, B. Schroer and J. Yngvason, String-localized quantum fields and modular localization, CMP 268 (2006) 621, math-ph/0511042

[38] Plaschke and J. Yngvason, Journal of Math. Phys. 53, (2012) 042301

[39] J. Mund, J. Math. Phys. 44 (2003) 2037

[40] B. Schroer, Unexplored regions in QFT and the conceptual foundations of the Standard Model, arXiv:1010.4431

[41] B. Schroer, An alternative to the gauge theory setting, Foun. of Phys. 41, (2011) 1543, arXiv:1012.0013

[42] J. Mund, Prog. Math. 251, (2007) 199, arXiv:hep-th/0502014

[43] J. Mund, contribution to the ICMP 2012

[44] S. Mandelstam, Ann. Phys. 19, (1962) 1

[45] G. Scharf, Quantum Gauge Theory, A True Ghost story, 2001, John Wiley\& Sons, INC.

[46] M. Gomes, J. H. Lowenstein, Nucl. Phys. B45, (1972) 252

[47] A.J. Swieca, Phys. Rev. D 15, (1975) 312

[48] B. Schroer, particle physics in the 60s and 70s and the legacy of contributions by J. A. Swieca, Eur.Phys.J.H 35, (2010) 53, arXiv:0712.0371

[49] P.W. Higgs, Phys. Rev. Lett. 12, (1964) 132 
[50] F. Englert and R. Brout, Phys. Rev. Lett. 13, (1964) 321

[51] G.S. Guralnik, C.R. Hagen and T.W.B. Kibble, Phys. Rev. Lett. 13 (1964) 585

[52] J. H. Lowenstein and B. Schroer, Phys. Rev. D7, (1975) 1929

[53] M. Duetsch, J. M. Gracia-Bondia, F- Scheck, J. C. Varilly, Quantum gauge models without classical Higgs mechanism, arXiv:1001.0932

[54] J. M. Gracia-Bondia, On the causal gauge principle, arXiv:0809.0160

[55] The mathematical backup of some of these ideas is part of an ongoing joint project with Jens Mund and Jakob Yngvason

[56] J. Bros and J. Mund, Braid group statistics implies scattering in threedimensional local quantum physics, Commun. Math. Phys. 315, (2012) 465 arXiv:1112.5785

[57] J. Yngvason, The Role of Type III Factors in Quantum Field Theory, Rept. Math. Phys. 55 (2005) 135, arXiv:math-ph/0411058

[58] R. Jost: TCP-Invarianz der Streumatrix und interpolierende Felder, Helvetica Phys. Acta 36, (1963) 77

[59] S. Hollands and R. Wald, Gen. Rel. Grav. 36 (2004) 2595

[60] B. Schroer, Studies in History and Philosophy of Modern Physics 41 (2010) 104-127, arXiv:0912.2874

[61] R. Kaehler and H.-P. Wiesbrock, Modular theory and the reconstruction of four-dimensional quantum field theories, Journal of Mathematical Physics 42, (2001) 74

[62] B. Schroer, The holistic structure of causal quantum theory, its implementation in the Einstein-Jordan conundrum and its violation in more recent particle theories, arXiv:1107.1374

[63] G. L. Sewell, Ann. Phys. 141 (1982) 201

[64] B. Schroer, Annals Phys. 275 (1999) 190

[65] B. Schroer, Pascual Jordan's legacy and the ongoing research in quantum field theory,

[66] D. Buchholz and K. Fredenhagen, Commun. Math. Phys. 56, (1977) 91

[67] S. Doplicher and J. E. Roberts, Why there is a field algebra with a compact gauge group describing the superselection structure in particle physics, Commun. Math. Phys. 131, (1990) 51-107

[68] K-H. Rehren and B. Schroer, Phys. Lett. B198, (1987) 84 
[69] B. Schroer, Space- and timelike superselection rules in conformal quantum field theories, hep-th/0010290 (2000), see also B. Schroer, Braided structure in 4-dimensional quantum field theory, Phys. Lett. B, 506, (2001) 337

[70] B. Schroer and J. A. Swieca, Phys. Rev. D 10, (1974) 480

[71] B. Schroer, J. A. Swieca and A. H. Voelkel, Phys. Rev. D 11, 1975

[72] N. M. Nikolov, K.-H. Rehren and I. Todorov, Commun. Math. Phys.279, (2008) 225

[73] A. A. Belavin, A. M. Polyakov and A. B. Zamolodchikov, Nucl. Phys. B 241 (2), (1984) 333

[74] N. Beisert, Nucl. Phys. B 682487 (2004) arXiv:hep-th/0310252

[75] K.-H. Rehren and B. Schroer, Einstein causality and Artin braids, Nucl. Phys. B 312, (1989) 715

[76] J. Fröhlich and F. Gabbiani, Braid statistics in local quantum theory, Rev. Math. Phys. 2, (1991) 251

[77] G. Mack, D-dimensional Conformal Field Theories with anomalous dimensions as Dual Resonance Models, arXiv:0909.1024

[78] G. Mack, D-independent representation of Conformal Field Theories in D dimensions via transformation to auxiliary Dual Resonance Models. Scalar amplitudes, arXiv:0907.2407v1

[79] Y. Kawahigashi and R. Longo, Commun. Math. Phys.244 (2004) 63

[80] B. Schroer, The holistic structure of causal quantum theory, its implementation in the Einstein-Jordan conundrum and its violation in more recent particle theories, arXiv:1107.1374

[81] R. Haag and B. Schroer, Postulates of Quantum Field Theory, J. Mat. Phys. 3, (1962) 248

[82] M. Dütsch and K.-H. Rehren, Ann. Henri Poincare 4, (2003) 613 